\title{
Integration of the Nonlinear Schroedinger Equation with a Self-Consistent Source
}

\section{V.K. Mel'nikov}

Laboratory of Theoretical Physics, Joint Institute for Nuclear Research, Head Post Office, P.O. Box 79, SU-101000 Moscow, USSR

\begin{abstract}
It is shown that the nonlinear Schroedinger equation with a selfconsistent source admits investigation by the inverse scattering method for the Dirac operator. The conditions are found under which the solutions of the nonlinear Schroedinger equation with a self-consistent source describe the creation and annihilation of solitons.
\end{abstract}

\section{Introduction}

At present, in the investigation of nonlinear evolution equations by the inverse scattering method there comes into view a new perspective trend: the case in point is the application of this method to integration of nonlinear evolution equations with a source. Being different in details, the use of the inverse scattering method for integrating different nonlinear evolution equations with a source has much in common both in the scheme of integration and in the dynamics of the obtained solutions. In this paper, these statements will be exemplified by the nonlinear Schroedinger equation with a self-consistent source. More precisely, we consider the integration of the following system of equations:

$$
\begin{array}{ll}
i \frac{\partial u}{\partial t}+2|u|^{2} u+\frac{\partial^{2} u}{\partial x^{2}} & =2 i \sum_{n=1}^{N}\left(\varphi_{n} p_{n}-\bar{\psi}_{n} \bar{q}_{n}\right), \\
\frac{\partial \varphi_{n}}{\partial x}+u \psi_{n}-i \zeta_{n} \varphi_{n}=\frac{\partial \psi_{n}}{\partial x}-\bar{u} \varphi_{n}+i \zeta_{n} \psi_{n}=0, & n=1, \ldots, N, N, \quad n=1, \ldots, N, \\
\frac{\partial p_{n}}{\partial x}+u q_{n}-i \zeta_{n} p_{n}=\frac{\partial q_{n}}{\partial x}-\bar{u} p_{n}+i \zeta_{n} q_{n}=0, \quad
\end{array}
$$

where the bar means complex conjugation. We shall assume that the function $u=u(x, t)$ at any $t \geq 0$ satisfies the requirement

$$
\sum_{r=0}^{2} \int_{-\infty}^{\infty}\left|\frac{\partial^{r} u(x, t)}{\partial x^{r}}\right| d x<\infty
$$


Taking this requirement into account the solutions $\varphi_{n}=\varphi_{n}(x, t), \psi_{n}=\psi_{n}(x, t)$ and $p_{n}=p_{n}(x, t), q_{n}=q_{n}(x, t)$ of Eqs. (2) and (3), respectively, are to be chosen so that the expression in the right-hand side of (1) for $t \geq 0$ should tend rapidly enough to zero if $x \rightarrow \pm \infty$. This can be done in two ways.

The first way of choosing solutions of Eqs. (2) and (3) is as follows. Let the quantities $\zeta_{1}, \ldots, \zeta_{N}$ lie in the upper half-plane of the complex parameter $\zeta$, i.e. $\operatorname{Im} \zeta_{n}>0, n=1, \ldots, N$. Let then the solution $\varphi_{n}, \psi_{n}$ of Eq. (2) tend to zero as $x \rightarrow-\infty$ and the solution $p_{n}, q_{n}$ of Eq. (3) tend to zero as $x \rightarrow \infty$, i.e. at any $t \geq 0$ the following asymptotics hold:

$$
\begin{array}{cl}
\left|\varphi_{n}(x, t)\right|+\left|\psi_{n}(x, t)\right| \rightarrow 0, & \text { if } \quad x \rightarrow-\infty, \\
\left|p_{n}(x, t)\right|+\left|q_{n}(x, t)\right| \rightarrow 0, & \text { if } \quad x \rightarrow \infty .
\end{array}
$$

As will be shown below, in this case the right-hand side of Eq. (1) for any $t \geq 0$ tends rapidly enough to zero if $x \rightarrow \pm \infty$ and the solution $u=u(x, t)$ satisfies the condition (4). In this case, the quantities $\zeta_{n}$ can be arbitrary functions of time $t$ for any $t \geq 0$ satisfying the condition $\operatorname{Im} \zeta_{n}>0, n=1, \ldots, N$. It appears that in this case the function $u=u(x, t)$ can describe a number of nontrivial processes, for instance, the decay and fusion of solitons, capture of solitons into an oscillatory regime of motion, and the formation of a bound state of several solitons.

The second way of choosing solutions of Eqs. (2) and (3) consists in the following. Let the quantities $\zeta_{1}, \ldots, \zeta_{N}$ be points of the discrete spectrum of the operator $L$ of the form

$$
L=\Lambda \partial+U, \quad \partial=\frac{\partial}{\partial x}
$$

where

$$
\Lambda=\operatorname{diag}(1,-1), \quad U=\left|\begin{array}{ll}
0 & u \\
\bar{u} & 0
\end{array}\right|,
$$

and lie as formerly in the upper half-plane $\operatorname{Im} \zeta>0$ of the complex parameter $\zeta$. In what follows, unless otherwise stated, we assume all points $\zeta=\zeta_{n}$ of the discrete spectrum of the operator $L$ to be simple, $n=1, \ldots, N$. Then, let the solution $\varphi_{n}, \psi_{n}$ of Eq. (2) tend to zero as $x \rightarrow \pm \infty$, i.e. it is obtained from the normalised to unity eigenfunction of the operator $L$ of the form (6), (7), which satisfies the eigenvalue $\zeta=\zeta_{n}$, by multiplying by the $x$ independent quantity; the solution $p_{n}, q_{n}$ of Eq. (3), on the contrary, tends to infinity as $x \rightarrow \pm \infty$, i.e. for any $t \geq 0$ the following asymptotics hold:

$$
\begin{array}{lll}
\left|\varphi_{n}(x, t)\right|+\left|\psi_{n}(x, t)\right| \rightarrow 0, & \text { if } & x \rightarrow \pm \infty, \\
\left|p_{n}(x, t)\right|+\left|q_{n}(x, t)\right| \rightarrow \infty, & \text { if } \quad x \rightarrow \pm \infty .
\end{array}
$$

As will be shown below, in this case the right-hand side of Eq. (1) for any $t \geq 0$ also tends rapidly enough to zero if $x \rightarrow \pm \infty$, and the solution $u=u(x, t)$ satisfies the condition (4). The quantities $\zeta_{n}$ turn out to be functions of time satisfying the condition

$$
\frac{d \zeta_{n}}{d t}=i W_{n}, \quad n=1, \ldots, N
$$

where the quantities $W_{n}$ are determined by the equality

$$
W_{n}=\varphi_{n}(x, t) q_{n}(x, t)-\psi_{n}(x, t) p_{n}(x, t),
$$


and by virtue of (2) and (3) are independent of $x$. Hence, it follows that the quantities $\zeta_{n}$ in the process of evolution can fall on the real axis from the upper half-plane of the complex parameter $\zeta$, i.e. the imaginary part $\operatorname{Im} \zeta_{n}$ of the quantity $\zeta_{n}$ can vanish at some time moment $t=t^{\prime}$. This leads to that in the process of evolution a soliton, corresponding to this eigenvalue, disappears, i.e. annihilates. Then, if for $t>t^{\prime}$ the quantity $\zeta_{n}$ goes away from the real axis, then a soliton that has disappeared appears again, i.e. is created. Thus, in this situation the function $u=u(x, t)$ can describe, apart from the afore-mentioned nontrivial processes, also the creation and annihilation of solitons.

The above-mentioned results are obtained by the inverse scattering method for the operator $L$ of the form (6), (7). Analogous results can be obtained for a modified Korteweg-de Vries equation with a self-consistent source, and after small changes of some details of this paper similar results can be obtained for the Liouville and sine-Gordon equations with self-consistent sources.

\section{Determining Relations}

The use of the inverse scattering method for integration of the system (1)-(3) is based on the following. We take the operator $L$ of the form (6), (7). Then, let the operator $A$ have the form [1],

$$
A=-i\left(2 \Lambda \partial^{2}+U \partial+\partial \cdot U+\Lambda U^{2}\right) .
$$

One can easily verify the validity of the equality

$$
[A, L]=-i\left(2 \Lambda U^{3}+\Lambda \frac{\partial^{2} U}{\partial x^{2}}\right),
$$

i.e.

$$
[A, L]=\left|\begin{array}{ll}
0 & \Delta \\
\bar{\Delta} & 0
\end{array}\right|,
$$

where

$$
\Delta=-i\left(2|u|^{2} u+\frac{\partial^{2} u}{\partial x^{2}}\right) .
$$

Now, let us consider the system of equations

$$
(L-i \zeta) f_{0}=0, \quad \frac{\partial f_{n}}{\partial x}=\tilde{\Psi}_{n} f_{0}, \quad n=1, \ldots, 2 N,
$$

with respect to unknown quantities $f_{0}, f_{1}, \ldots, f_{2 N}$. Here and everywhere below the tilde " " means transposition, i.e., in particular, the transition from the vector-column to the vector-row. We shall assume that $f_{0}$ is the second order square matrix, $\Psi_{1}, \ldots, \Psi_{2 N}$ are vector-columns with two components each, and consequently, $f_{1}, \ldots, f_{2 N}$ are two-component vector-rows. Using the solution $f_{0}$, $f_{1}, \ldots, f_{2 N}$ of the system (14) we determine the quantities $g_{0}, g_{1}, \ldots, g_{2 N}$ by the equalities

$$
\begin{aligned}
& g_{0}=\frac{\partial f_{0}}{\partial t}+A f_{0}+\sum_{n=1}^{2 N} \Phi_{n} f_{n}, \\
& g_{n}=\tilde{\Psi}_{n} \Lambda f_{0}-i\left(\zeta-\zeta_{n}\right) f_{n}, \quad n=1, \ldots, 2 N,
\end{aligned}
$$


where $\Phi_{1}, \ldots, \Phi_{2 N}$ are vector-columns with two components each, and consequently, $g_{0}$ is the second order square matrix, and $g_{1}, \ldots, g_{2 N}$ are two-component vector-rows. Now, let us find out what requirements are to be imposed on the matrix $U$ and vectors $\Phi_{1}, \ldots, \Phi_{2 N}, \Psi_{1}, \ldots, \Psi_{2 N}$ so that the quantities determined above $g_{0}, g_{1}, \ldots, g_{2 N}$ should satisfy the relations

$$
(L-i \zeta) g_{0}=\sum_{n=1}^{2 N} \Phi_{n} g_{n}, \quad \frac{\partial g_{n}}{\partial x} \equiv 0, \quad n=1, \ldots, 2 N
$$

After simple calculations one can easily verify that for the validity of these relations it is necessary and sufficient to fulfill the conditions

$$
\begin{aligned}
\frac{\partial L}{\partial t}+[A, L] & =\sum_{n=1}^{2 N}\left[\Lambda, \Phi_{n} \tilde{\Psi}_{n}\right], \\
\left(L-i \zeta_{n}\right) \Phi_{n} & =\left(\tilde{L}-i \zeta_{n}\right) \Psi_{n}=0, \quad n=1, \ldots, 2 N,
\end{aligned}
$$

where

$$
\tilde{L}=-\Lambda \partial+\tilde{U}
$$

Now we take the matrix $\sigma$ of the form

$$
\sigma=\left|\begin{array}{ll}
0 & 1 \\
1 & 0
\end{array}\right|
$$

One can easily verify that by virtue of (7) the equalities $\sigma \Lambda \sigma=-\Lambda$, and $\sigma U \sigma=\tilde{U}$ hold, i.e. taking account of (6) and (18) we get the equality $\sigma L \sigma=\tilde{L}$. Hence, it follows that if the vector-column $\Phi=\Phi_{0}$ satisfies the equation

$$
(L-i \zeta) \Phi=0
$$

at $\zeta=\zeta_{0}$, then the vector-column $\Psi=\sigma \Phi_{0}$ satisfies the equation

$$
(\tilde{L}-i \zeta) \Psi=0
$$

at the same value of the parameter $\zeta=\zeta_{0}$. Then, let $E=\Lambda \sigma$. According to (7) and (19) the equalities $E \Lambda \tilde{E}=-\Lambda$ and $E U \tilde{E}=-\tilde{U}=-\tilde{U}$ are valid, i.e. according to (6) and (18) we get that $E L \tilde{E}=-\bar{L}, E \tilde{L} \tilde{E}=-L^{*}$, where $\bar{L}=\Lambda \partial+\bar{U}$, $L^{*}=-\Lambda \partial+U$. Thus, we get that if the vector-column $\Phi=\Phi_{0}$ satisfies Eq. (20) at $\zeta=\zeta_{0}$, then the vector-column $\Phi=E \bar{\Phi}_{0}$ satisfies the same equation but at $\zeta=\bar{\zeta}_{0}$. Moreover, if the vector-column $\Psi=\Psi_{0}$ satisfies Eq. (21) at $\zeta=\zeta_{0}$, then the vector-column $\Psi=E \bar{\Psi}_{0}$ also satisfies Eq. (21) but at $\zeta=\bar{\zeta}_{0}$. From the afore-said we assume that at $n=1, \ldots, N$,

$$
\Phi_{n}=\left|\begin{array}{c}
\varphi_{n} \\
\psi_{n}
\end{array}\right|, \quad \Psi_{n}=\left|\begin{array}{c}
q_{n} \\
p_{n}
\end{array}\right|, \quad n=1, \ldots, N .
$$

Finally, we assume that at $n=1, \ldots, N$ the condition $\zeta_{N+n}=\bar{\zeta}_{n}$ is fulfilled, and according to this condition we assume that

$$
\Phi_{N+n}=\left|\begin{array}{c}
\bar{\psi}_{n} \\
-\bar{\varphi}_{n}
\end{array}\right|, \quad \Psi_{N+n}=\left|\begin{array}{c}
\bar{p}_{n} \\
-\bar{q}_{n}
\end{array}\right|, \quad n=1, \ldots, N .
$$

Hence, there follows the equality

$$
\sum_{n=1}^{2 N}\left[\Lambda, \Phi_{n} \tilde{\Psi}_{n}\right]=2\left|\begin{array}{ll}
0 & \gamma \\
\bar{\gamma} & 0
\end{array}\right|
$$


where

$$
\gamma=\sum_{n=1}^{N}\left(\varphi_{n} p_{n}-\bar{\psi}_{n} \bar{q}_{n}\right)
$$

Thus, if the vector-columns $\Phi_{n}$ and $\Psi_{n}$ of the form (22) satisfy, respectively, Eqs. (20) and (21) at $\zeta=\zeta_{n}$, then their components $\varphi_{n}, \psi_{n}$ and $p_{n}, q_{n}$ satisfy, respectively, Eqs. (2) and (3), $n=1, \ldots, N$. Then, the vector-columns $\Phi_{N+n}$ and $\Psi_{N+n}$ determined by (23) satisfy, according to the afore-said, Eqs. (20) and (21), respectively, at $\zeta=\zeta_{N+n}=\bar{\zeta}_{n}, n=1, \ldots, N$. Finally, by virtue of (6), (7), (12), (13), (24) and (25) the first equation of the system (17) is equivalent to (1). This means that relations (16) under the above choice of the vectors $\Phi_{n}$ and $\Psi_{n}$, $n=1, \ldots, 2 N$, are equivalent to the system of Eqs. (1)-(3).

A remarkable property of relations (16) is the fact that they can be used to derive evolution equations for all scattering data of the operator $L$ of the form (6), (7) with the potential $u=u(x, t)$ satisfying the system (1)-(3). This allows one to call relations (16) the determining relations. Note that the method of determining relations has first been used in [2] to integrate the Kortewegde Vries equation with a self-consistent source. Somewhat later [3] it has been shown that this method can be used to integrate many other nonlinear evolution equations with a self-consistent source, especially, to integrate the nonlinear Schroedinger equation with a self-consistent source.

\section{Auxiliary Statements}

This section contains auxiliary statements about the properties of the solutions of Eq. (20) formulated in a form convenient for using in the present paper.

Thus, let $f_{0}^{-}$and $f_{0}^{+}$be matrix solutions of the equation

$$
(L-i \zeta) f_{0}=0
$$

at any $\zeta \in(-\infty, \infty)$ having the asymptotics

$$
\begin{aligned}
& f_{0}^{-} \sim \exp (i \zeta \Lambda x), \quad \text { if } \quad x \rightarrow-\infty \\
& f_{0}^{+} \sim \exp (i \zeta \Lambda x), \quad \text { if } \quad x \rightarrow \infty
\end{aligned}
$$

One can easily verify that the matrices $f_{0}^{-}$and $f_{0}^{+}$at any $\zeta \in(-\infty, \infty)$ satisfy the integral equations

$$
\begin{aligned}
& f_{0}^{-}=\exp (i \zeta \Lambda x)-\int_{-\infty}^{x} \exp [i \zeta \Lambda(x-z)] \Lambda U(z) f_{0}^{-}(z, \zeta) d z \\
& f_{0}^{+}=\exp (i \zeta \Lambda x)+\int_{x}^{\infty} \exp [i \zeta \Lambda(x-z)] \Lambda U(z) f_{0}^{+}(z, \zeta) d z .
\end{aligned}
$$

Assume that

$$
f_{0}^{-}=\left|\begin{array}{ll}
\varphi_{1}^{-} & \varphi_{2}^{-} \\
\psi_{1}^{-} & \psi_{2}^{-}
\end{array}\right|, \quad f_{0}^{+}=\left|\begin{array}{cc}
\varphi_{1}^{+} & \varphi_{2}^{+} \\
\psi_{1}^{+} & \psi_{2}^{+}
\end{array}\right|
$$


Substituting these expressions into equalities (28) we can easily find that between $\varphi_{1}^{-}$and $\psi_{1}^{-}$there is a connection of the form

$$
\begin{aligned}
& \varphi_{1}^{-}=\exp (i \zeta x)-\int_{-\infty}^{x} \exp [i \zeta(x-z)] u(z) \psi_{1}^{-}(z, \zeta) d z \\
& \psi_{1}^{-}=\int_{-\infty}^{x} \exp [-i \zeta(x-z)] \bar{u}(z) \varphi_{1}^{-}(z, \zeta) d z
\end{aligned}
$$

and an analogous connection between $\varphi_{2}^{-}$and $\psi_{2}^{-}$

$$
\begin{aligned}
& \varphi_{2}^{-}=-\int_{-\infty}^{x} \exp [i \zeta(x-z)] u(z) \psi_{2}^{-}(z, \zeta) d z, \\
& \psi_{2}^{-}=\exp (-i \zeta x)+\int_{-\infty}^{x} \exp [-i \zeta(x-z)] \bar{u}(z) \varphi_{2}^{-}(z, \zeta) d z
\end{aligned}
$$

Moreover, between $\varphi_{1}^{+}$and $\psi_{1}^{+}$a relation is fulfilled of the form

$$
\begin{aligned}
& \varphi_{1}^{+}=\exp (i \zeta x)+\int_{x}^{\infty} \exp [i \zeta(x-z)] u(z) \psi_{1}^{+}(z, \zeta) d z \\
& \psi_{1}^{+}=-\int_{x}^{\infty} \exp [-i \zeta(x-z)] \bar{u}(z) \varphi_{1}^{+}(z, \zeta) d z,
\end{aligned}
$$

and an analogous relation between $\varphi_{2}^{+}$and $\psi_{2}^{+}$

$$
\begin{aligned}
& \varphi_{2}^{+}=\int_{x}^{\infty} \exp [i \zeta(x-z)] u(z) \psi_{2}^{+}(z, \zeta) d z \\
& \psi_{2}^{+}=\exp (-i \zeta x)-\int_{x}^{\infty} \exp [-i \zeta(x-z)] \bar{u}(z) \varphi_{2}^{+}(z, \zeta) d z
\end{aligned}
$$

For any $\zeta \in(-\infty, \infty)$ for solving Eqs. (30)-(33) we apply the method of successive approximations. The solutions of Eq. (26) thus obtained have a number of remarkable properties. To describe these properties we assume that

$$
\begin{aligned}
& \varphi_{1}^{-}(x, \zeta)=\hat{\varphi}_{1}^{-}(x, \zeta) \exp (i \zeta x), \\
& \psi_{1}^{-}(x, \zeta)=\hat{\psi}_{1}^{-}(x, \zeta) \exp (i \zeta x), \\
& \varphi_{2}^{+}(x, \zeta)=\hat{\varphi}_{2}^{+}(x, \zeta) \exp (-i \zeta x), \\
& \psi_{2}^{+}(x, \zeta)=\hat{\psi}_{2}^{+}(x, \zeta) \exp (-i \zeta x)
\end{aligned}
$$


Substituting these expressions into equalities (30) and (33) we derive the following integral equations:

$$
\begin{aligned}
& \hat{\varphi}_{1}^{-}(x, \zeta)=1-\int_{-\infty}^{x} u(z) \hat{\psi}_{1}^{-}(z, \zeta) d z, \\
& \hat{\psi}_{1}^{-}(x, \zeta)=\int_{-\infty}^{x} \exp [-2 i \zeta(x-z)] \bar{u}(z) \hat{\varphi}_{1}^{-}(z, \zeta) d z, \\
& \hat{\varphi}_{2}^{+}(x, \zeta)=\int_{x}^{\infty} \exp [2 i \zeta(x-z)] u(z) \hat{\psi}_{2}^{+}(z, \zeta) d z, \\
& \hat{\psi}_{2}^{+}(x, \zeta)=1-\int_{x}^{\infty} \bar{u}(z) \hat{\varphi}_{2}^{+}(z, \zeta) d z
\end{aligned}
$$

It follows from these equations that the functions $\hat{\varphi}_{1}^{-}, \hat{\psi}_{1}^{-}$and $\hat{\varphi}_{2}^{+}, \hat{\psi}_{2}^{+}$admit an analytic continuation in $\zeta$ into the lower half-plane $\operatorname{Im} \zeta<0$. Moreover, in the closed half-plane $\operatorname{Im} \zeta \leq 0$ the following asymptotics are valid:

$$
\begin{array}{lll}
\hat{\varphi}_{1}^{-}(x, \zeta) \rightarrow 1, & \hat{\psi}_{1}^{-}(x, \zeta) \rightarrow 0, & \text { if } \quad x \rightarrow-\infty \\
\hat{\varphi}_{2}^{+}(x, \zeta) \rightarrow 0, & \hat{\psi}_{2}^{+}(x, \zeta) \rightarrow 1, & \text { if } \quad x \rightarrow \infty .
\end{array}
$$

By virtue of (34) this means that in the closed half-plane $\operatorname{Im} \zeta \leq 0$ the following equalities hold:

$$
\begin{aligned}
\lim _{x \rightarrow-\infty}\left[\varphi_{1}^{-}(x, \zeta) \exp (-i \zeta x)\right] & =\lim _{x \rightarrow \infty}\left[\psi_{2}^{+}(x, \zeta) \exp (i \zeta x)\right]=1 \\
\lim _{x \rightarrow-\infty}\left[\psi_{1}^{-}(x, \zeta) \exp (-i \zeta x)\right] & =\lim _{x \rightarrow \infty}\left[\varphi_{2}^{+}(x, \zeta) \exp (i \zeta x)\right]=0 .
\end{aligned}
$$

Thus, at any $\zeta$ belonging to the lower half-plane $\operatorname{Im} \zeta<0$ the solution $\varphi_{1}^{-}$, $\psi_{1}^{-}$exponentially decreases as $x \rightarrow-\infty$ and the solution $\varphi_{2}^{+}, \psi_{2}^{+}$exponentially decreases as $x \rightarrow \infty$. Then, we assume that

$$
\begin{aligned}
& \varphi_{2}^{-}(x, \zeta)=\hat{\varphi}_{2}^{-}(x, \zeta) \exp (-i \zeta x) \\
& \psi_{2}^{-}(x, \zeta)=\hat{\psi}_{2}^{-}(x, \zeta) \exp (-i \zeta x), \\
& \varphi_{1}^{+}(x, \zeta)=\hat{\varphi}_{1}^{+}(x, \zeta) \exp (i \zeta x), \\
& \psi_{1}^{+}(x, \zeta)=\hat{\psi}_{1}^{+}(x, \zeta) \exp (i \zeta x)
\end{aligned}
$$


Upon substituting these expressions into equalities (31) and (32) we get the following integral equations:

$$
\begin{aligned}
& \hat{\varphi}_{2}^{-}(x, \zeta)=-\int_{-\infty}^{x} \exp [2 i \zeta(x-z)] u(z) \hat{\psi}_{2}^{-}(z, \zeta) d z, \\
& \hat{\psi}_{2}^{-}(x, \zeta)=1+\int_{-\infty}^{x} \bar{u}(z) \hat{\varphi}_{2}^{-}(z, \zeta) d z, \\
& \hat{\varphi}_{1}^{+}(x, \zeta)=1+\int_{x}^{\infty} u(z) \hat{\psi}_{1}^{+}(z, \zeta) d z, \\
& \hat{\psi}_{1}^{+}(x, \zeta)=-\int_{x}^{\infty} \exp [-2 i \zeta(x-z)] \bar{u}(z) \hat{\varphi}_{1}^{+}(z, \zeta) d z .
\end{aligned}
$$

It follows from these equations that the functions $\hat{\varphi}_{2}^{-}, \hat{\psi}_{2}^{-}$and $\hat{\varphi}_{1}^{+}, \hat{\psi}_{1}^{+}$admit an analytic continuation in $\zeta$ into the upper half-plane $\operatorname{Im} \zeta>0$. Moreover, in the closed half-plane $\operatorname{Im} \zeta \geq 0$ there hold the asymptotics

$$
\begin{array}{ll}
\hat{\varphi}_{2}^{-}(x, \zeta) \rightarrow 0, & \hat{\psi}_{2}^{-}(x, \zeta) \rightarrow 1, \quad \text { if } \quad x \rightarrow-\infty, \\
\hat{\varphi}_{1}^{+}(x, \zeta) \rightarrow 1, \quad \hat{\psi}_{1}^{+}(x, \zeta) \rightarrow 0, & \text { if } \quad x \rightarrow \infty .
\end{array}
$$

With allowance made for (38) this means that in the closed half-plane $\operatorname{Im} \zeta \geq 0$ the following equalities are valid:

$$
\begin{aligned}
& \lim _{x \rightarrow-\infty}\left[\varphi_{2}^{-}(x, \zeta) \exp (i \zeta x)\right]=\lim _{x \rightarrow \infty}\left[\psi_{1}^{+}(x, \zeta) \exp (-i \zeta x)\right]=0, \\
& \lim _{x \rightarrow-\infty}\left[\psi_{2}^{-}(x, \zeta) \exp (i \zeta x)\right]=\lim _{x \rightarrow \infty}\left[\varphi_{1}^{+}(x, \zeta) \exp (-i \zeta x)\right]=1 .
\end{aligned}
$$

Thus, at any $\zeta$ in the upper half-plane $\operatorname{Im} \zeta>0$ the solution $\varphi_{2}^{-}, \psi_{2}^{-}$exponentially decreases as $x \rightarrow-\infty$ and the solution $\varphi_{1}^{+}, \psi_{1}^{+}$exponentially decreases as $x \rightarrow \infty$.

For any $\zeta \in(-\infty, \infty)$ there holds the equality

$$
f_{0}^{+}(x, \zeta)=f_{0}^{-}(x, \zeta) S(\zeta)
$$

where the elements $S_{\alpha \beta}(\zeta)$ of the matrix $S(\zeta)$ are independent of $x, \alpha, \beta=1,2$. Using (27), (29), and (42) we easily find that for any $\zeta \in(-\infty, \infty)$ there hold the equalities

$$
\begin{aligned}
& S_{11}(\zeta)=\varphi_{1}^{+}(x, \zeta) \psi_{2}^{-}(x, \zeta)-\psi_{1}^{+}(x, \zeta) \varphi_{2}^{-}(x, \zeta), \\
& S_{12}(\zeta)=\varphi_{2}^{+}(x, \zeta) \psi_{2}^{-}(x, \zeta)-\psi_{2}^{+}(x, \zeta) \varphi_{2}^{-}(x, \zeta), \\
& S_{21}(\zeta)=-\varphi_{1}^{+}(x, \zeta) \psi_{1}^{-}(x, \zeta)+\psi_{1}^{+}(x, \zeta) \varphi_{1}^{-}(x, \zeta), \\
& S_{22}(\zeta)=-\varphi_{2}^{+}(x, \zeta) \psi_{1}^{-}(x, \zeta)+\psi_{2}^{+}(x, \zeta) \varphi_{1}^{-}(x, \zeta)
\end{aligned}
$$

From the afore-said these equalities result in that the function $S_{11}(\zeta)$ admits an analytic continuation in $\zeta$ into the upper half-plane $\operatorname{Im} \zeta>0$ and the function $S_{22}(\zeta)$ admits an analytic continuation in $\zeta$ into the lower half-plane $\operatorname{Im} \zeta<0$. To zeros $\zeta=\zeta_{n}$ of the function $S_{11}(\zeta)$ in the upper half-plane $\operatorname{Im} \zeta>0$ there correspond points of the discrete spectrum of the operator $L$ as, according to (43), at $\zeta=\zeta_{n}$ the equalities

$$
\varphi_{1}^{+}\left(x, \zeta_{n}\right)=B_{n} \varphi_{2}^{-}\left(x, \zeta_{n}\right), \quad \psi_{1}^{+}\left(x, \zeta_{n}\right)=B_{n} \psi_{2}^{-}\left(x, \zeta_{n}\right)
$$


are valid where the quantities $B_{n}$ are independent of $x$. Analogously, to zeros $\zeta=\hat{\zeta}_{n}$ of the function $S_{22}(\zeta)$ in the lower half-plane $\operatorname{Im} \zeta<0$ there also correspond points of the discrete spectrum of the operator $L$ since on the basis of (43) at $\zeta=\hat{\zeta}_{n}$ there hold the equalities

$$
\varphi_{2}^{+}\left(x, \hat{\zeta}_{n}\right)=\hat{B}_{n} \varphi_{1}^{-}\left(x, \hat{\zeta}_{n}\right), \quad \psi_{2}^{+}\left(x, \hat{\zeta}_{n}\right)=\hat{B}_{n} \psi_{1}^{-}\left(x, \hat{\zeta}_{n}\right),
$$

where the quantities $\hat{B}_{n}$ are also independent of $x$.

By virtue of (26), (27), and (29) at any real $\zeta$ there hold the equalities

$$
\begin{array}{ll}
\varphi_{2}^{-}(x, \zeta)=-\bar{\psi}_{1}^{-}(x, \zeta), & \psi_{2}^{-}(x, \zeta)=\bar{\varphi}_{1}^{-}(x, \zeta), \\
\varphi_{2}^{+}(x, \zeta)=-\bar{\psi}_{1}^{+}(x, \zeta), & \psi_{2}^{+}(x, \zeta)=\bar{\varphi}_{1}^{+}(x, \zeta) .
\end{array}
$$

With allowance for (43) it follows that at any real $\zeta$ the equalities

$$
\begin{aligned}
& S_{11}(\zeta)=\varphi_{1}^{+}(x, \zeta) \bar{\varphi}_{1}^{-}(x, \zeta)+\psi_{1}^{+}(x, \zeta) \bar{\psi}_{1}^{-}(x, \zeta), \\
& S_{12}(\zeta)=\bar{\varphi}_{1}^{+}(x, \zeta) \bar{\psi}_{1}^{-}(x, \zeta)-\bar{\psi}_{1}^{+}(x, \zeta) \bar{\varphi}_{1}^{-}(x, \zeta), \\
& S_{21}(\zeta)=-\varphi_{1}^{+}(x, \zeta) \psi_{1}^{-}(x, \zeta)+\psi_{1}^{+}(x, \zeta) \varphi_{1}^{-}(x, \zeta), \\
& S_{22}(\zeta)=\bar{\varphi}_{1}^{+}(x, \zeta) \varphi_{1}^{-}(x, \zeta)+\bar{\psi}_{1}^{+}(x, \zeta) \psi_{1}^{-}(x, \zeta)
\end{aligned}
$$

are valid, i.e. at any real $\zeta$ the following relations hold:

$$
S_{22}(\zeta)=\bar{S}_{11}(\zeta), \quad S_{21}(\zeta)=-\bar{S}_{12}(\zeta)
$$

Thus, according to (27) and (42) at any real $\zeta$ there holds the equality

$$
\operatorname{det} S(\zeta)=\left|S_{11}(\zeta)\right|^{2}+\left|S_{12}(\zeta)\right|^{2}=1
$$

Then, at any $\zeta$ in the upper half-plane $\operatorname{Im} \zeta>0$ by virtue of (26), (27), and (29) the equalities

$$
\begin{array}{ll}
\bar{\varphi}_{2}^{-}(x, \zeta)=-\psi_{1}^{-}(x, \bar{\zeta}), & \bar{\psi}_{2}^{-}(x, \zeta)=\varphi_{1}^{-}(x, \bar{\zeta}), \\
\varphi_{2}^{+}(x, \bar{\zeta})=-\bar{\psi}_{1}^{+}(x, \zeta), & \psi_{2}^{+}(x, \bar{\zeta})=\bar{\varphi}_{1}^{+}(x, \bar{\zeta})
\end{array}
$$

are valid. Based on these equalities and taking account of (43) we get that

$$
\bar{S}_{11}(\zeta)=\varphi_{1}^{-}(x, \bar{\zeta}) \psi_{2}^{+}(x, \bar{\zeta})-\psi_{1}^{-}(x, \bar{\zeta}) \varphi_{2}^{+}(x, \bar{\zeta}) .
$$

Comparing this equality with the one resulting from (43)

$$
S_{22}(\bar{\zeta})=\varphi_{1}^{-}(x, \bar{\zeta}) \psi_{2}^{+}(x, \bar{\zeta})-\psi_{1}^{-}(x, \bar{\zeta}) \varphi_{2}^{+}(x, \bar{\zeta})
$$

we verify that at any $\zeta$ in the upper half-plane $\operatorname{Im} \zeta>0$ the following relation holds:

$$
\bar{S}_{11}(\zeta)=S_{22}(\bar{\zeta}), \quad \operatorname{Im} \zeta>0 .
$$

Hence, it follows that to each zero $\zeta=\zeta_{n}$ of the function $S_{11}(\zeta)$ in the upper half-plane $\operatorname{Im} \zeta>0$ there corresponds the zero $\zeta=\hat{\zeta}_{n}$ of the function $S_{22}(\zeta)$ in the lower half-plane $\operatorname{Im} \zeta<0$ such that $\hat{\zeta}_{n}=\bar{\zeta}_{n}$. Then, according to equalities (44), (45), and (47) we find that between the quantities $B_{n}$ and $\hat{B}_{n}$ there is a relation $\hat{B}=-\bar{B}_{n}$.

Using the integral equations (35) we easily verify that at any $\zeta$ in the lower half-plane $\operatorname{Im} \zeta<0$ there are asymptotics

$$
\begin{array}{ll}
\hat{\psi}_{1}^{-}(x, \zeta) \rightarrow 0, & \text { if } \quad x \rightarrow \infty, \\
\hat{\varphi}_{2}^{+}(x, \zeta) \rightarrow 0, & \text { if } \quad x \rightarrow-\infty .
\end{array}
$$


By virtue of (34), (36), and (43) it follows that at any $\zeta$ in the lower half-plane $\operatorname{Im} \zeta<0$ the asymptotics

$$
\begin{aligned}
& \hat{\varphi}_{1}^{-}(x, \zeta) \rightarrow S_{22}(\zeta), \quad \text { if } \quad x \rightarrow \infty, \\
& \hat{\psi}_{2}^{+}(x, \zeta) \rightarrow S_{22}(\zeta), \quad \text { if } \quad x \rightarrow-\infty,
\end{aligned}
$$

are valid. Thus, based on (34) we get that at any $\zeta$ in the lower half-plane $\operatorname{Im} \zeta<0$ the following equalities hold:

$$
\begin{aligned}
& \lim _{x \rightarrow \infty}\left[\varphi_{1}^{-}(x, \zeta) \exp (-i \zeta x)\right]=\lim _{x \rightarrow-\infty}\left[\psi_{2}^{+}(x, \zeta) \exp (i \zeta x)\right]=S_{22}(\zeta), \\
& \lim _{x \rightarrow \infty}\left[\psi_{1}^{-}(x, \zeta) \exp (-i \zeta x)\right]=\lim _{x \rightarrow-\infty}\left[\varphi_{2}^{+}(x, \zeta) \exp (i \zeta x)\right]=0 .
\end{aligned}
$$

Analogously, using the integral equations (39) we find that at any $\zeta$ in the upper half-plane $\operatorname{Im} \zeta>0$ there hold asymptotics

$$
\begin{aligned}
& \hat{\varphi}_{2}^{-}(x, \zeta) \rightarrow 0, \quad \text { if } \quad x \rightarrow \infty . \\
& \hat{\psi}_{1}^{+}(x, \zeta) \rightarrow 0, \quad \text { if } \quad x \rightarrow-\infty .
\end{aligned}
$$

With (38), (40), and (43) taken into account it follows that at any $\zeta$ in the upper half-plane $\operatorname{Im} \zeta>0$ the following asymptotics hold:

$$
\begin{aligned}
& \hat{\psi}_{2}^{-}(x, \zeta) \rightarrow S_{11}(\zeta), \quad \text { if } \quad x \rightarrow \infty, \\
& \hat{\varphi}_{1}^{+}(x, \zeta) \rightarrow S_{11}(\zeta), \quad \text { if } \quad x \rightarrow-\infty .
\end{aligned}
$$

Thus, according to (38) we find that at any $\zeta$ in the upper half-plane $\operatorname{Im} \zeta>0$ the equalities

$$
\begin{aligned}
& \lim _{x \rightarrow \infty}\left[\varphi_{2}^{-}(x, \zeta) \exp (i \zeta x)\right]=\lim _{x \rightarrow-\infty}\left[\psi_{1}^{+}(x, \zeta) \exp (-i \zeta x)\right]=0 \\
& \lim _{x \rightarrow \infty}\left[\psi_{2}^{-}(x, \zeta) \exp (i \zeta x)\right]=\lim _{x \rightarrow-\infty}\left[\varphi_{1}^{+}(x, \zeta) \exp (-i \zeta x)\right]=S_{11}(\zeta)
\end{aligned}
$$

are fulfilled.

\section{Evolution Equation for the $S$-Matrix in the Case of a Source Satisfying the Conditions (5)}

Now we proceed to deriving evolution equations for the scattering data of the operator $L$ of the form (6), (7) with the potential $u=u(x, t)$ satisfying the system (1)-(3). However, to avoid cumbersome formulae, we shall derive these equations first for the case of a source satisfying the conditions (5) and then for the case of a source satisfying the conditions (8).

Now, we take arbitrarily $N$ points $\zeta=\zeta_{n}, n=1, \ldots, N$, in the upper half-plane $\operatorname{Im} \zeta>0$, and accordig to equalities (22) assume at $n=1, \ldots, N$ that

$$
\begin{aligned}
& \Phi_{n}^{-}=\left|\begin{array}{l}
\varphi_{n}^{-}(x) \\
\psi_{n}^{-}(x)
\end{array}\right|=\alpha_{n}^{-}\left|\begin{array}{c}
\varphi_{1}^{+}\left(x, \zeta_{n}\right) \\
\psi_{1}^{+}\left(x, \zeta_{n}\right)
\end{array}\right|, \\
& \Psi_{n}^{-}=\left|\begin{array}{l}
q_{n}^{-}(x) \\
p_{n}^{-}(x)
\end{array}\right|=\beta_{n}^{-}\left|\begin{array}{l}
\psi_{2}^{-}\left(x, \zeta_{n}\right) \\
\varphi_{2}^{-}\left(x, \zeta_{n}\right)
\end{array}\right|, \\
& \Phi_{n}^{+}=\left|\begin{array}{l}
\varphi_{n}^{+}(x) \\
\psi_{n}^{+}(x)
\end{array}\right|=\alpha_{n}^{+}\left|\begin{array}{l}
\varphi_{2}^{-}\left(x, \zeta_{n}\right) \\
\psi_{2}^{-}\left(x, \zeta_{n}\right)
\end{array}\right|, \\
& \Psi_{n}^{+}=\left|\begin{array}{l}
q_{n}^{+}(x) \\
p_{n}^{+}(x)
\end{array}\right|=\beta_{n}^{+}\left|\begin{array}{l}
\psi_{1}^{+}\left(x, \zeta_{n}\right) \\
\varphi_{1}^{+}\left(x, \zeta_{n}\right)
\end{array}\right|,
\end{aligned}
$$


where the quantities $\alpha_{n}^{-}, \beta_{n}^{-}$and $\alpha_{n}^{+}, \beta_{n}^{+}$are independent of $x$. Then, taking account of (23) and (47) we assume at $n=1, \ldots, N$ that

$$
\begin{gathered}
\Phi_{N+n}^{-}=\left|\begin{array}{c}
\bar{\psi}_{n}^{-}(x) \\
\bar{\varphi}_{n}^{-}(x)
\end{array}\right|=\bar{\alpha}_{n}^{-}\left|\begin{array}{c}
\bar{\psi}_{1}^{+}\left(x, \zeta_{n}\right) \\
-\bar{\varphi}_{1}^{+}\left(x, \zeta_{n}\right)
\end{array}\right|=-\bar{\alpha}_{n}^{-}\left|\begin{array}{c}
\varphi_{2}^{+}\left(x, \bar{\zeta}_{n}\right) \\
\psi_{2}^{+}\left(x, \bar{\zeta}_{n}\right)
\end{array}\right|, \\
\Psi_{N+n}^{-}=\left|\begin{array}{c}
\bar{p}_{n}^{-}(x) \\
-\bar{q}_{n}^{-}(x)
\end{array}\right|=\bar{\beta}_{n}^{-}\left|\begin{array}{c}
\bar{\varphi}_{2}^{-}\left(x, \zeta_{n}\right) \\
-\bar{\psi}_{2}^{-}\left(x, \zeta_{n}\right)
\end{array}\right|=-\bar{\beta}_{n}^{-}\left|\begin{array}{c}
\psi_{1}^{-}\left(x, \bar{\zeta}_{n}\right) \\
\varphi_{1}^{-}\left(x, \bar{\zeta}_{n}\right)
\end{array}\right|, \\
\Phi_{N+n}^{+}=\left|\begin{array}{c}
\bar{\psi}_{n}^{+}(x) \\
-\bar{\varphi}_{n}^{+}(x)
\end{array}\right|=\bar{\alpha}_{n}^{+}\left|\begin{array}{c}
\bar{\psi}_{2}^{-}\left(x, \zeta_{n}\right) \\
-\bar{\varphi}_{2}^{-}\left(x, \zeta_{n}\right)
\end{array}\right|=\bar{\alpha}_{n}^{+}\left|\begin{array}{c}
\varphi_{1}^{-}\left(x, \bar{\zeta}_{n}\right) \\
\psi_{1}^{-}\left(x, \bar{\zeta}_{n}\right)
\end{array}\right|, \\
\Psi_{N+n}^{+}=\left|\begin{array}{c}
\bar{p}_{n}^{+}(x) \\
-\bar{q}_{n}^{+}(x)
\end{array}\right|=\bar{\beta}_{n}^{+}\left|\begin{array}{c}
\bar{\varphi}_{1}^{+}\left(x, \zeta_{n}\right) \\
-\bar{\psi}_{1}^{+}\left(x, \zeta_{n}\right)
\end{array}\right|=\bar{\beta}_{n}^{+}\left|\begin{array}{c}
\psi_{2}^{+}\left(x, \bar{\zeta}_{n}\right) \\
\varphi_{2}^{+}\left(x, \bar{\zeta}_{n}\right)
\end{array}\right| .
\end{gathered}
$$

According to (25) and (51)-(54) the quantities

$$
\gamma^{-}=\sum_{n=1}^{N}\left(\varphi_{n}^{-} p_{n}^{-}-\bar{\psi}_{n}^{-} \bar{q}_{n}^{-}\right), \quad \gamma^{+}=\sum_{n=1}^{N}\left(\varphi_{n}^{+} p_{n}^{+}-\bar{\psi}_{n}^{+} \bar{q}_{n}^{+}\right)
$$

can be represented as

$$
\begin{aligned}
& \gamma^{-}=\sum_{n=1}^{N}\left[c_{n}^{-} \varphi_{1}^{+}\left(x, \zeta_{n}\right) \varphi_{2}^{-}\left(x, \zeta_{n}\right)+\bar{c}_{n}^{-} \varphi_{1}^{-}\left(x, \bar{\zeta}_{n}\right) \varphi_{2}^{+}\left(x, \bar{\zeta}_{n}\right)\right], \\
& \gamma^{+}=\sum_{n=1}^{N}\left[c_{n}^{+} \varphi_{1}^{+}\left(x, \zeta_{n}\right) \varphi_{2}^{-}\left(x, \zeta_{n}\right)+\bar{c}_{n}^{+} \varphi_{1}^{-}\left(x, \bar{\zeta}_{n}\right) \varphi_{2}^{+}\left(x, \bar{\zeta}_{n}\right)\right],
\end{aligned}
$$

where $c_{n}^{-}=\alpha_{n}^{-} \beta_{n}^{-}$and $c_{n}^{+}=\alpha_{n}^{+} \beta_{n}^{+}, n=1, \ldots, N$. By virtue of (37), (41), (49), and (50) it follows that $\left|\gamma^{-}(x)\right|+\left|\gamma^{+}(x)\right| \rightarrow 0$ as $x \rightarrow \pm \infty$. Moreover, if $c_{n}^{-}=c_{n}^{+}=c_{n}$ at $n=1, \ldots, N$, then we have identically $\gamma^{-}(x) \equiv \gamma^{+}(x)$. This means that two possibilities we have for deriving determining relations for the system (1)-(3) finally lead to the same result if we will not violate the condition $c_{n}^{-}=c_{n}^{+}=c_{n}$, $n=1, \ldots, N$. With this remark in mind we assume at $n=1, \ldots, 2 N$ that

$$
f_{n}^{-}=\int_{-\infty}^{x} \tilde{\Psi}_{n}^{-}(z) f_{0}^{-}(z, \zeta) d z, \quad f_{n}^{+}=-\int_{x}^{\infty} \tilde{\Psi}_{n}^{+}(z) f_{0}^{+}(z, \zeta) d z .
$$

It follows from these equalities that $f_{n}^{-}$and $f_{n}^{+}$at $n=1, \ldots, 2 N$ are vectorrows, respectively, with two components $f_{n, 1}^{-}, f_{n, 2}^{-}$and $f_{n, 1}^{+}, f_{n, 2}^{+}$each. Obviously, by virtue of (37) and (41) the components $f_{n, 1}^{-}$and $f_{n, 2}^{+}$admits an analytic continuation in $\zeta$ into the lower half-plane $\operatorname{Im} \zeta<0$ and the components $f_{n, 2}^{-}$ and $f_{n, 1}^{+}$admit an analytic continuation in $\zeta$ into the upper half-plane $\operatorname{Im} \zeta>0$, $n=1, \ldots, 2 N$.

Finally, according to equalities (15) we assume that

$$
\begin{aligned}
& g_{0}^{-}=\frac{\partial f_{0}^{-}}{\partial t}+A f_{0}^{-}+\sum_{n=1}^{2 N} \Phi_{n}^{-} f_{n}^{-}, \\
& g_{n}^{-}=\tilde{\Psi}_{n}^{-} \Lambda f_{0}^{-}-i\left(\zeta-\zeta_{n}\right) f_{n}^{-}, \quad n=1, \ldots, 2 N,
\end{aligned}
$$




$$
\begin{aligned}
& g_{0}^{+}=\frac{\partial f_{0}^{+}}{\partial t}+A f_{0}^{+}+\sum_{n=1}^{2 N} \Phi_{n}^{+} f_{n}^{+}, \\
& g_{n}^{+}=\tilde{\Psi}_{n}^{+} \Lambda f_{0}^{+}-i\left(\zeta-\zeta_{n}\right) f_{n}^{+}, \quad n=1, \ldots, 2 N .
\end{aligned}
$$

Of course, we assume that an $n=1, \ldots, N$ the relation $\zeta_{N+n}=\bar{\zeta}_{n}$ is fulfilled. Based on (37), (41), and (55) one can easily verify that the first column of the matrix $g_{0}^{-}$and the last column of the matrix $g_{0}^{+}$admit an analytic continuation in $\zeta$ into the lower half-plane $\operatorname{Im} \zeta<0$, and the last column of the matrix $g_{0}^{-}$ and the first column of the matrix $g_{0}^{+}$admit an analytic continuation in $\zeta$ into the upper half-plane $\operatorname{Im} \zeta>0$. Moreover, one can easily see that $g_{n}^{-}$and $g_{n}^{+}$at $n=1, \ldots, 2 N$ are vector-rows, respectively, with two components $g_{n, 1}^{-}, g_{n, 2}^{-}$and $g_{n, 1}^{+}, g_{n, 2}^{+}$each. According to (37), (41), and (55) the components $g_{n, 1}^{-}$and $g_{n, 2}^{+}$ admit an analytic continuation in $\zeta$ into the lower half-plane $\operatorname{Im} \zeta<0$ and the components $g_{n, 2}^{-}$and $g_{n, 1}^{+}$admit an analytic continuation in $\zeta$ into the upper halfplane $\operatorname{Im} \zeta>0, n=1, \ldots, 2 N$. From equalities (41) and (51)-(54) $n=1, \ldots, 2 N$ there follow asymptotics

$$
\begin{aligned}
& \left\|\Psi_{n}^{-}(x)\right\| \rightarrow 0, \quad \text { if } \quad x \rightarrow-\infty, \\
& \left\|\Psi_{n}^{+}(x)\right\| \rightarrow 0, \quad \text { if } \quad x \rightarrow \infty .
\end{aligned}
$$

Hence, by virtue of (55)-(57) it follows that at any $\zeta \in(-\infty, \infty)$ and $n=1, \ldots, 2 N$ the asymptotics

$$
\begin{aligned}
& \left\|f_{n}^{-}(x, \zeta)\right\|+\left\|g_{n}^{-}(x, \zeta)\right\| \rightarrow 0, \quad \text { if } \quad x \rightarrow-\infty \\
& \left\|f_{n}^{+}(x, \zeta)\right\|+\left\|g_{n}^{+}(x, \zeta)\right\| \rightarrow 0, \quad \text { if } \quad x \rightarrow \infty
\end{aligned}
$$

are valid. Thus, taking account of identities $\frac{\partial g_{n}^{-}}{\partial x}=\frac{\partial g_{n}^{+}}{\partial x} \equiv 0, n=1, \ldots, 2 N$, we get that at $x, \zeta \in(-\infty, \infty)$ the following identities hold:

$$
g_{n}^{-}(x, \zeta)=g_{n}^{+}(x, \zeta) \equiv 0, \quad n=1, \ldots, 2 N .
$$

This means that in accordance with relations (16) the matrices $g_{0}^{-}$and $g_{0}^{+}$at any real $\zeta$ satisfy the equations

$$
(L-i \zeta) g_{0}^{-}=(L-i \zeta) g_{0}^{+}=0 .
$$

According to (4), (11), and (51)-(57) there follow the equalities

$$
\begin{aligned}
& g_{0}^{-}(x, \zeta)=f_{0}^{-}(x, \zeta)\left(2 i \zeta^{2} \Lambda+C_{0}^{-}\right), \\
& g_{0}^{+}(x, \zeta)=f_{0}^{+}(x, \zeta)\left(2 i \zeta^{2} \Lambda+C_{0}^{+}\right),
\end{aligned}
$$

where

$$
\begin{aligned}
& C_{0}^{-}=\operatorname{diag}\left(-i \sum_{n=1}^{N} c_{n}^{-} \frac{S_{11}\left(\zeta_{n}\right)}{\zeta-\zeta_{n}}, i \sum_{n=1}^{N} \bar{c}_{n}^{-} \frac{S_{22}\left(\bar{\zeta}_{n}\right)}{\zeta-\bar{\zeta}_{n}}\right), \\
& C_{0}^{+}=\operatorname{diag}\left(-i \sum_{n=1}^{N} \bar{c}_{n}^{+} \frac{S_{22}\left(\bar{\zeta}_{n}\right)}{\zeta-\bar{\zeta}_{n}}, i \sum_{n=1}^{N} c_{n}^{+} \frac{S_{11}\left(\zeta_{n}\right)}{\zeta-\zeta_{n}}\right) .
\end{aligned}
$$

Now consider the matrix $G_{0}$ of the form

$$
G_{0}=g_{0}^{+}(x, \zeta)-g_{0}^{-}(x, \zeta) S(\zeta) .
$$


Based on (42) and (60) we find that

$$
G_{0}=f_{0}^{-}(x, \zeta)\left\{-2 i \zeta^{2}[\Lambda, S(\zeta)]-C_{0}^{-} S(\zeta)+S(\zeta) C_{0}^{+}\right\}
$$

On the other hand, by virtue of (42), (56), and (57) the equality

$$
\begin{aligned}
g_{0}^{+}(x, \zeta)= & g_{0}^{-}(x, \zeta) S(\zeta)+f_{0}^{-}(x, \zeta) \frac{\partial S(\zeta)}{\partial t} \\
& +\sum_{n=1}^{2 N}\left[\Phi_{n}^{+}(x) f_{n}^{+}(x, \zeta)-\Phi_{n}^{-}(x) f_{n}^{-}(x, \zeta) S(\zeta)\right]
\end{aligned}
$$

holds. Using equalities (37), (41), (42), (50), (51), (53), and (55) we can verify that at any $\zeta \in(-\infty, \infty), n=1, \ldots, N$ and $x \rightarrow \infty$ the following asymptotics are valid:

$$
\begin{aligned}
\Phi_{n}^{-}(x) f_{n}^{-}(x, \zeta) S(\zeta) & \sim i P c_{n}^{-} \frac{S_{11}\left(\zeta_{n}\right)}{\zeta-\zeta_{n}} \exp (i \zeta x), \\
\Phi_{N+n}^{-}(x) f_{N+n}^{-}(x, \zeta) S(\zeta) & \sim i Q \bar{c}_{n}^{-} \frac{S_{22}\left(\bar{\zeta}_{n}\right)}{\zeta-\bar{\zeta}_{n}} \exp (-i \zeta x),
\end{aligned}
$$

where $P=\operatorname{diag}(-1,0)$ and $Q=\operatorname{diag}(0,1)$. Then, taking account of equalities (37), (41), (42), (50), (52), (54), and (55) we are convinced that at any $\zeta \in(-\infty, \infty)$, $n=1, \ldots, N$ and $x \rightarrow \infty$ there hold the asymptotics

$$
\begin{aligned}
\Phi_{n}^{+}(x) f_{n}^{+}(x, \zeta) & \sim i Q c_{n}^{+} \frac{S_{11}\left(\zeta_{n}\right)}{\zeta-\zeta_{n}} \exp (-i \zeta x), \\
\Phi_{N+n}^{+}(x) f_{N+n}^{+}(x, \zeta) & \sim i P \bar{c}_{n}^{+} \frac{S_{22}\left(\bar{\zeta}_{n}\right)}{\zeta-\bar{\zeta}_{n}} \exp (i \zeta x) .
\end{aligned}
$$

According to (59), (61), and (64) there follows the equality

$$
\begin{aligned}
& \sum_{n=1}^{2 N}\left[\Phi_{n}^{+}(x) f_{n}^{+}(x, \zeta)-\Phi_{n}^{-}(x) f_{n}^{-}(x, \zeta) S(\zeta)\right] \\
& \quad=f_{0}^{+}(x, \zeta)\left(C_{0}^{+}-C_{0}^{-}\right)=f_{0}^{-}(x, \zeta) S(\zeta)\left(C_{0}^{+}-C_{0}^{-}\right)
\end{aligned}
$$

Based on this equality relation (64) becomes

$$
g_{0}^{+}(x, \zeta)=g_{0}^{-}(x, \zeta) S(\zeta)+f_{0}^{-}(x, \zeta)\left[\frac{\partial S(\zeta)}{\partial t}+S(\zeta)\left(C_{0}^{+}-C_{0}^{-}\right)\right]
$$

It follows that the quantity $G_{0}$ determined by (62) admits the representation

$$
G_{0}=f_{0}^{-}(x, \zeta)\left[\frac{\partial S(\zeta)}{\partial t}+S(\zeta)\left(C_{0}^{+}-C_{0}^{-}\right)\right] .
$$

Comparing this equality with (63) we immediately find that the evolution equation for the $S$-matrix has the form

$$
\frac{\partial S(\zeta)}{\partial t}+\left[2 i \zeta^{2} \Lambda+C_{0}^{-}, S(\zeta)\right]=0,
$$


i.e.

$$
\begin{aligned}
& \frac{\partial S_{11}(\zeta)}{\partial t}=\frac{\partial S_{22}(\zeta)}{\partial t}=0, \\
& \frac{\partial S_{12}(\zeta)}{\partial t}+\left\{4 i \zeta^{2}-i \sum_{n=1}^{N}\left[c_{n}^{-} \frac{S_{11}\left(\zeta_{n}\right)}{\zeta-\zeta_{n}}+\bar{c}_{n}^{-} \frac{S_{22}\left(\bar{\zeta}_{n}\right)}{\zeta-\bar{\zeta}_{n}}\right]\right\} S_{12}(\zeta)=0, \\
& \frac{\partial S_{21}(\zeta)}{\partial t}-\left\{4 i \zeta^{2}-i \sum_{n=1}^{N}\left[c_{n}^{-} \frac{S_{11}\left(\zeta_{n}\right)}{\zeta-\zeta_{n}}+\bar{c}_{n}^{-} \frac{S_{22}\left(\bar{\zeta}_{n}\right)}{\zeta-\bar{\zeta}_{n}}\right]\right\} S_{21}(\zeta)=0 .
\end{aligned}
$$

It is easily seen that by virtue of these equations the diagonal elements of the $S$-matrix are independent of time $t$. This implies that in the case of a source satisfying the conditions (5) the points of the discrete spectrum of the operator $L$ of the form (6), (7) are also independent of time if the potential $u=u(x, t)$ satisfies the system (1)-(3). Then, with (48) taken into account we get that relations (46) will be fulfilled at any $\zeta \in(-\infty, \infty)$ and $t>0$, if they are valid at any $\zeta \in(-\infty, \infty)$ and $t=0$. Finally, if the points $\zeta=\zeta_{n}$ are the points of the discrete spectrum of the operator $L$, i.e. $S_{11}\left(\zeta_{n}\right)=S_{22}\left(\bar{\zeta}_{n}\right)=0, n=1, \ldots, N$, then equations for the elements $S_{12}(\zeta)$ and $S_{21}(\zeta)$ become

$$
\frac{\partial S_{12}(\zeta)}{\partial t}+4 i \zeta^{2} S_{12}(\zeta)=\frac{\partial S_{21}(\zeta)}{\partial t}-4 i \zeta^{2} S_{21}(\zeta)=0
$$

i.e., in this case the evolution equations for the elements of the $S$-matrix coincide with those that are valid for the nonlinear Schroedinger equation without a source [1].

\section{Evolution Equations for the Normalisation Constants in the Case of a Source Satisfying the Conditions (5)}

Let $\sigma_{r}^{-}$and $\sigma_{r}^{+}$be vector-columns formed, respectively, by the elements of the $r^{\text {th }}$ column of the matrice $f_{0}^{-}$and $f_{0}^{+}$of the form (29), $r=1,2$. Then, let $\tau_{r}^{-}$and $\tau_{r}^{+}$be vector-columns formed, respectively, by the elements of the $r^{\text {th }}$ column of the matrices $g_{0}^{-}$and $g_{0}^{+}$of the form (56) and (57), $r=1$,2. Finally, let $\zeta=\zeta_{m}^{\prime}$ be zeros of the function $S_{11}(\zeta)$ in the upper half-plane $\operatorname{Im} \zeta>0, m=1, \ldots, m_{0}$. According to equalities (44) and (45) we assume that at $m=1, \ldots, m_{0}$

$$
\begin{aligned}
& G_{m}=\tau_{1}^{+}\left(x, \zeta_{m}^{\prime}\right)-B_{m} \tau_{2}^{-}\left(x, \zeta_{m}^{\prime}\right), \\
& \hat{G}_{m}=\tau_{2}^{+}\left(x, \bar{\zeta}_{m}^{\prime}\right)-\hat{B}_{m} \tau_{1}^{-}\left(x, \bar{\zeta}_{m}^{\prime}\right) .
\end{aligned}
$$

According to equalities (60) and (61) we have

$$
\begin{aligned}
& \tau_{1}^{+}\left(x, \zeta_{m}^{\prime}\right)=\left(2 i \zeta_{m}^{\prime 2}-i \sum_{n=1}^{N} \bar{c}_{n}^{+} \frac{S_{22}\left(\bar{\zeta}_{n}\right)}{\zeta_{m}^{\prime}-\bar{\zeta}_{n}}\right) \sigma_{1}^{+}\left(x, \zeta_{m}^{\prime}\right), \\
& \tau_{2}^{-}\left(x, \zeta_{m}^{\prime}\right)=-\left(2 i \zeta_{m}^{\prime 2}-i \sum_{n=1}^{N} \bar{c}_{n}^{-} \frac{S_{22}\left(\bar{\zeta}_{n}\right)}{\zeta_{m}^{\prime}-\bar{\zeta}_{n}}\right) \sigma_{2}^{-}\left(x, \zeta_{m}^{\prime}\right), \\
& \tau_{2}^{+}\left(x, \bar{\zeta}_{m}^{\prime}\right)=-\left(2 i \bar{\zeta}_{m}^{\prime 2}-i \sum_{n=1}^{N} c_{n}^{+} \frac{S_{11}\left(\zeta_{n}\right)}{\bar{\zeta}_{m}^{\prime}-\zeta_{n}}\right) \sigma_{2}^{+}\left(x, \bar{\zeta}_{m}^{\prime}\right), \\
& \tau_{1}^{-}\left(x, \bar{\zeta}_{m}^{\prime}\right)=\left(2 i \bar{\zeta}_{m}^{\prime 2}-i \sum_{n=1}^{N} c_{n}^{-} \frac{S_{11}\left(\zeta_{n}\right)}{\bar{\zeta}_{m}^{\prime}-\zeta_{n}}\right) \sigma_{1}^{-}\left(x, \bar{\zeta}_{m}^{\prime}\right) .
\end{aligned}
$$


From equalities (44) and (45) it follows that the quantities $G_{m}$ and $\hat{G}_{m}$ determined by (66) can be written in the following form:

$$
\begin{aligned}
& G_{m}=\left(4 i \zeta_{m}^{\prime 2}-2 i \sum_{n=1}^{N} \bar{c}_{n} \frac{S_{22}\left(\bar{\zeta}_{n}\right)}{\zeta_{m}^{\prime}-\bar{\zeta}_{n}}\right) B_{m} \sigma_{2}^{-}\left(x, \zeta_{m}^{\prime}\right), \\
& \hat{G}_{m}=-\left(4 i \bar{\zeta}_{m}^{\prime 2}-2 i \sum_{n=1}^{N} c_{n} \frac{S_{11}\left(\zeta_{n}\right)}{\bar{\zeta}_{m}^{\prime}-\zeta_{n}}\right) \hat{B}_{m} \sigma_{1}^{-}\left(x, \bar{\zeta}_{m}^{\prime}\right),
\end{aligned}
$$

where $c_{n}=c_{n}^{-}=c_{n}^{+}, n=1, \ldots, N$.

On the other hand, by virtue of (44) and (45) the equalities

$$
\begin{aligned}
& \frac{\partial \sigma_{1}^{+}\left(x, \zeta_{m}^{\prime}\right)}{\partial t}=B_{m} \frac{\partial \sigma_{2}^{-}\left(x, \zeta_{m}^{\prime}\right)}{\partial t}+\frac{\partial B_{m}}{\partial t} \sigma_{2}^{-}\left(x, \zeta_{m}^{\prime}\right), \\
& \frac{\partial \sigma_{2}^{+}\left(x, \bar{\zeta}_{m}^{\prime}\right)}{\partial t}=\hat{B}_{m} \frac{\partial \sigma_{1}^{-}\left(x, \bar{\zeta}_{m}^{\prime}\right)}{\partial t}+\frac{\partial \hat{B}_{m}}{\partial t} \sigma_{1}^{-}\left(x, \bar{\zeta}_{m}^{\prime}\right)
\end{aligned}
$$

hold. Using these equalities, from (44), (45), and (55)-(57) we find that

$$
\begin{aligned}
\tau_{1}^{+}\left(x, \zeta_{m}^{\prime}\right)= & B_{m} \tau_{2}^{-}\left(x, \zeta_{m}^{\prime}\right)-\sum_{n=1}^{2 N}\left[\Phi_{n}^{-}(x) \int_{-\infty}^{x} \tilde{\Psi}_{n}^{-}(z) \sigma_{2}^{-}\left(z, \zeta_{m}^{\prime}\right) d z\right. \\
& \left.+\Phi_{n}^{+}(x) \int_{x}^{\infty} \tilde{\Psi}_{n}^{+}(z) \sigma_{2}^{-}\left(z, \zeta_{m}^{\prime}\right) d z\right] B_{m}+\frac{\partial B_{m}}{\partial t} \sigma_{2}^{-}\left(x, \zeta_{m}^{\prime}\right) \\
\tau_{2}^{+}\left(x, \bar{\zeta}_{m}^{\prime}\right)= & \hat{B}_{m} \tau_{1}^{-}\left(x, \bar{\zeta}_{m}^{\prime}\right)-\sum_{n=1}^{2 N}\left[\Phi_{n}^{-}(x) \int_{-\infty}^{x} \tilde{\Psi}_{n}^{-}(z) \sigma_{1}^{-}\left(z, \bar{\zeta}_{m}^{\prime}\right) d z\right. \\
& \left.+\Phi_{n}^{+}(x) \int_{x}^{\infty} \tilde{\Psi}_{n}^{+}(z) \sigma_{1}^{-}\left(z, \bar{\zeta}_{m}^{\prime}\right) d z\right] \hat{B}_{m}+\frac{\partial \hat{B}_{m}}{\partial t} \sigma_{1}^{-}\left(x, \bar{\zeta}_{m}^{\prime}\right)
\end{aligned}
$$

According to (51)-(54) at $m=1, \ldots, m_{0}$ and $n=1, \ldots, 2 N$ the equalities

$$
\begin{aligned}
i\left(\zeta_{m}^{\prime}-\zeta_{n}\right) \int_{-\infty}^{x} \tilde{\Psi}_{n}^{-}(z) \sigma_{2}^{-}\left(z, \zeta_{m}^{\prime}\right) d z & =\tilde{\Psi}_{n}^{-}(x) \Lambda \sigma_{2}^{-}\left(x, \zeta_{m}^{\prime}\right), \\
i\left(\zeta_{m}^{\prime}-\zeta_{n}\right) \int_{x}^{\infty} \tilde{\Psi}_{n}^{+}(z) \sigma_{2}^{-}\left(z, \zeta_{m}^{\prime}\right) d z & =-\tilde{\Psi}_{n}^{+}(x) \Lambda \sigma_{2}^{-}\left(x, \zeta_{m}^{\prime}\right), \\
i\left(\bar{\zeta}_{m}^{\prime}-\zeta_{n}\right) \int_{-\infty}^{x} \tilde{\Psi}_{n}^{-}(z) \sigma_{1}^{-}\left(z, \bar{\zeta}_{m}^{\prime}\right) d z & =\tilde{\Psi}_{n}^{-}(x) \Lambda \sigma_{1}^{-}\left(x, \bar{\zeta}_{m}^{\prime}\right), \\
i\left(\bar{\zeta}_{m}^{\prime}-\zeta_{n}\right) \int_{x}^{\infty} \tilde{\Psi}_{n}^{+}(z) \sigma_{1}^{-}\left(z, \bar{\zeta}_{m}^{\prime}\right) d z & =-\tilde{\Psi}_{n}^{+}(x) \Lambda \sigma_{1}^{-}\left(x, \bar{\zeta}_{m}^{\prime}\right)
\end{aligned}
$$


hold. Of course, we assume that $\zeta_{N+n}=\bar{\zeta}_{n}, n=1, \ldots, N$. Then, in accordance with (43) and (51)-(54) at $n=1, \ldots, N$ there hold the equalities

$$
\begin{aligned}
\Phi_{n}^{-}(x) \tilde{\Psi}_{n}^{-}(x)-\Phi_{n}^{+}(x) \tilde{\Psi}_{n}^{+}(x) & =c_{n} S_{11}\left(\zeta_{n}\right) \Lambda, \\
\Phi_{N+n}^{-}(x) \tilde{\Psi}_{N+n}^{-}(x)-\Phi_{N+n}^{+}(x) \tilde{\Psi}_{N+n}^{+}(x) & =-\bar{c}_{n} S_{22}\left(\bar{\zeta}_{n}\right) \Lambda .
\end{aligned}
$$

With these equalities taken into account relations (68) become

$$
\begin{aligned}
\tau_{1}^{+}\left(x, \zeta_{m}^{\prime}\right)= & B_{m} \tau_{2}^{-}\left(x, \zeta_{m}^{\prime}\right)+\frac{\partial B_{m}}{\partial t} \sigma_{2}^{-}\left(x, \zeta_{m}^{\prime}\right) \\
& +i \sum_{n=1}^{N}\left[c_{n} \frac{S_{11}\left(\zeta_{n}\right)}{\zeta_{m}^{\prime}-\zeta_{n}}-\bar{c}_{n} \frac{S_{22}\left(\bar{\zeta}_{n}\right)}{\zeta_{m}^{\prime}-\bar{\zeta}_{n}}\right] B_{m} \sigma_{2}^{-}\left(x, \zeta_{m}^{\prime}\right), \\
\tau_{2}^{+}\left(x, \bar{\zeta}_{m}^{\prime}\right)= & \hat{B}_{m} \tau_{1}^{-}\left(x, \bar{\zeta}_{m}^{\prime}\right)+\frac{\partial \hat{B}_{m}}{\partial t} \sigma_{1}^{-}\left(x, \bar{\zeta}_{m}^{\prime}\right) \\
& +i \sum_{n=1}^{N}\left[c_{n} \frac{S_{11}\left(\zeta_{n}\right)}{\bar{\zeta}_{m}^{\prime}-\zeta_{n}}-\bar{c}_{n} \frac{S_{22}\left(\bar{\zeta}_{n}\right)}{\bar{\zeta}_{m}^{\prime}-\bar{\zeta}_{n}}\right] \hat{B}_{m} \sigma_{1}^{-}\left(x, \bar{\zeta}_{m}^{\prime}\right)
\end{aligned}
$$

Hence, it follows that the quantities $G_{m}$ and $\hat{G}_{m}$ determined by (66) admit the representation

$$
\begin{aligned}
& G_{m}=\left\{\frac{\partial B_{m}}{\partial t}+i \sum_{n=1}^{N}\left[c_{n} \frac{S_{11}\left(\zeta_{n}\right)}{\zeta_{m}^{\prime}-\zeta_{n}}-\bar{c}_{n} \frac{S_{22}\left(\bar{\zeta}_{n}\right)}{\zeta_{m}^{\prime}-\bar{\zeta}_{n}}\right] B_{m}\right\} \sigma_{2}^{-}\left(x, \zeta_{m}^{\prime}\right), \\
& \hat{G}_{m}=\left\{\frac{\partial \hat{B}_{m}}{\partial t}+i \sum_{n=1}^{N}\left[c_{n} \frac{S_{11}\left(\zeta_{n}\right)}{\bar{\zeta}_{m}^{\prime}-\zeta_{n}}-\bar{c}_{n} \frac{S_{22}\left(\bar{\zeta}_{n}\right)}{\bar{\zeta}_{m}^{\prime}-\bar{\zeta}_{n}}\right] \hat{B}_{m}\right\} \sigma_{1}^{-}\left(x, \bar{\zeta}_{m}^{\prime}\right) .
\end{aligned}
$$

Comparing these equalities with (67) we immediately get that the evolution equations for the normalisation constants $B_{m}$ and $\hat{B}_{m}$ have the form

$$
\begin{aligned}
& \frac{\partial B_{m}}{\partial t}-\left\{4 i \zeta_{m}^{\prime 2}-i \sum_{n=1}^{N}\left[c_{n} \frac{S_{11}\left(\zeta_{n}\right)}{\zeta_{m}^{\prime}-\zeta_{n}}+\bar{c}_{n} \frac{S_{22}\left(\bar{\zeta}_{n}\right)}{\zeta_{m}^{\prime}-\bar{\zeta}_{n}}\right]\right\} B_{m}=0, \\
& \frac{\partial \hat{B}_{m}}{\partial t}+\left\{4 i \bar{\zeta}_{m}^{\prime 2}-i \sum_{n=1}^{N}\left[c_{n} \frac{S_{11}\left(\zeta_{n}\right)}{\bar{\zeta}_{m}^{\prime}-\zeta_{n}}+\bar{c}_{n} \frac{S_{22}\left(\bar{\zeta}_{n}\right)}{\bar{\zeta}_{m}^{\prime}-\zeta_{n}}\right]\right\} \hat{B}_{m}=0 .
\end{aligned}
$$

Comparing these equations with (65) for the elements of the $S$-matrix, one can easily verify that the evolution equation for the quantity $B_{m}$ results from the equation for $S_{21}(\zeta)$ if one assumes in it $\zeta=\zeta_{m}^{\prime}$, and the evolution equation for the quantity $\hat{B}_{m}$ follows from the equation for $S_{12}(\zeta)$ if one assumes in it $\zeta=\bar{\zeta}_{m}^{\prime}$, $m=1, \ldots, m_{0}$. Then, it follows from eqs. (69) that if the quantities $B_{m}$ and $\hat{B}_{m}$ at $t=0$ satisfy the relation $\hat{B}_{m}=-\bar{B}_{m}$, this relation will be fulfilled at all $t>0$. Finally, at $m_{0}=N$ in Eqs. (69) one can assume that $\zeta_{n}=\zeta_{n}^{\prime}+\delta_{n}, n=1, \ldots, N$, and pass to the limit at $\delta_{n} \rightarrow 0$. As a result we derive the equations

$$
\begin{aligned}
& \frac{\partial B_{m}}{\partial t}-\left[4 i \zeta_{m}^{\prime 2}+i c_{m} S_{11}^{\prime}\left(\zeta_{m}^{\prime}\right)\right] B_{m}=0, \\
& \frac{\partial \hat{B}_{m}}{\partial t}+\left[4 i \bar{\zeta}_{m}^{\prime 2}+i \bar{c}_{m} S_{22}^{\prime}\left(\bar{\zeta}_{m}^{\prime}\right)\right] \hat{B}_{m}=0 .
\end{aligned}
$$


The system of Eqs. (65) and (69) describes the evolution in time of all scattering data necessary for solving the inverse scattering problem for the operator $L$ of the form (6), (7). Thus, in the case of a source satisfying the conditions (5) solving of the Cauchy problem for the system (1)-(3) is reduced to finding a solution of the direct and inverse scattering problem for the operator $L$ of the form (6), (7).

\section{Evolution Equation for the $S$-Matrix in the Case of a Source Satisfying the Conditions (8)}

Now, we consider another way of choosing solutions of the linear system (2), (3), forming a source in the right-hand side of Eq. (1). Let $\zeta=\zeta_{n}$ be zeros of the function $S_{11}(\zeta)$ in the upper half-plane $\operatorname{Im} \zeta>0, n=1, \ldots, N$. In accordance with equalities (22) and (23) we assume that at $n=1, \ldots, N$,

$$
\begin{array}{ccc}
\Phi_{n}=\left|\begin{array}{c}
\varphi_{n}(x) \\
\psi_{n}(x)
\end{array}\right|, & \Psi_{n}=\left|\begin{array}{c}
q_{n}(x) \\
p_{n}(x)
\end{array}\right|=c_{n}\left|\begin{array}{l}
\psi_{1}^{+}\left(x, \zeta_{n}\right) \\
\varphi_{1}^{+}\left(x, \zeta_{n}\right)
\end{array}\right|, \\
\Phi_{N+n}=\left|\begin{array}{c}
\bar{\psi}_{n}(x) \\
-\bar{\varphi}_{n}(x)
\end{array}\right|, & \Psi_{N+n}=\left|\begin{array}{c}
\bar{p}_{n}(x) \\
-\bar{q}_{n}(x)
\end{array}\right|=\bar{c}_{n}\left|\begin{array}{c}
\bar{\varphi}_{1}^{+}\left(x, \zeta_{n}\right) \\
-\bar{\psi}_{1}^{+}\left(x, \zeta_{n}\right)
\end{array}\right| \\
& =\bar{c}_{n}\left|\begin{array}{c}
\psi_{2}^{+}\left(x, \bar{\zeta}_{n}\right) \\
\varphi_{2}^{+}\left(x, \bar{\zeta}_{n}\right)
\end{array}\right|,
\end{array}
$$

where the quantities $c_{n}$ are independent of $x$, and the solution $\varphi_{n}(x), \psi_{n}(x)$ of Eq. (2) satisfies the condition

$$
\varphi_{n}(x) \psi_{1}^{+}\left(x, \zeta_{n}\right)-\psi_{n}(x) \varphi_{1}^{+}\left(x, \zeta_{n}\right) \equiv 1, \quad n=1, \ldots, N .
$$

From (41) and (44) it follows that the solution $\varphi_{n}(x), \psi_{n}(x)$ of Eq. (2) has asymptotics

$$
\begin{aligned}
B_{n} \varphi_{n}(x) \exp \left(-i \zeta_{n} x\right) & \rightarrow 1, \\
\psi_{n}(x) \exp \left(-i \zeta_{n} x\right) & \rightarrow 0, \quad \text { if } \quad x \rightarrow-\infty, \\
\varphi_{n}(x) \exp \left(i \zeta_{n} x\right) & \rightarrow 0, \\
\psi_{n}(x) \exp \left(i \zeta_{n} x\right) & \rightarrow-1, \quad \text { if } \quad x \rightarrow \infty .
\end{aligned}
$$

According to (70) the quantiy $\gamma$ determined by (25) admits the representation

$$
\gamma=\sum_{n=1}^{N}\left[c_{n} \varphi_{n}(x) \varphi_{1}^{+}\left(x, \zeta_{n}\right)-\bar{c}_{n} \bar{\psi}_{n}(x) \bar{\psi}_{1}^{+}\left(x, \zeta_{n}\right)\right] .
$$

By virtue of (41), (44), and (72) it follows that $\gamma(x) \rightarrow 0$ at $x \rightarrow \pm \infty$.

Now, we assume that at $n=1, \ldots, 2 N$,

$$
f_{n}^{-}=\int_{-\infty}^{x} \tilde{\Psi}_{n}(z) f_{0}^{-}(z, \zeta) d z, \quad f_{n}^{+}=-\int_{x}^{\infty} \tilde{\Psi}_{n}(z) f_{0}^{+}(z, \zeta) d z
$$


Then, we assume

$$
\begin{aligned}
& g_{0}^{-}=\frac{\partial f_{0}^{-}}{\partial t}+A f_{0}^{-}+\sum_{n=1}^{2 N} \Phi_{n} f_{n}^{-}, \\
& g_{n}^{-}=\tilde{\Psi}_{n} \Lambda f_{0}^{-}-i\left(\zeta-\zeta_{n}\right) f_{n}^{-}, \quad n=1, \ldots, 2 N, \\
& g_{0}^{+}=\frac{\partial f_{0}^{+}}{\partial t}+A f_{0}^{+}+\sum_{n=1}^{2 N} \Phi_{n} f_{n}^{+}, \\
& g_{n}^{+}=\tilde{\Psi}_{n} \Lambda f_{0}^{+}-i\left(\zeta-\zeta_{n}\right) f_{n}^{+}, \quad n=1, \ldots, 2 N,
\end{aligned}
$$

where $\zeta_{N+n}=\bar{\zeta}_{n}, n=1, \ldots, N$. One can easily see that the quantities, determined by equalities (73) and (74), have the properties similar to those that have been detected earlier for the quantities determined by (55)-(57). Indeed, taking account of (44) and (70) we find that at $n=1, \ldots, 2 N$ the asymptotics are valid

$$
\left\|\Psi_{n}(x)\right\| \rightarrow 0, \quad \text { if } x \rightarrow \pm \infty .
$$

Hence, it follows that the quantities determined by (74) satisfy equalities (58) and (59). Then, by virtue of (4), (11), (59), (70), and (72)-(74) we find that the equalities

$$
g_{0}^{-}(x, \zeta)=f_{0}^{-}(x, \zeta)\left(2 i \zeta^{2} \Lambda+C^{-}\right), \quad g_{0}^{+}(x, \zeta)=f_{0}^{+}(x, \zeta)\left(2 i \zeta^{2} \Lambda+C^{+}\right)
$$

are valid where

$$
\begin{aligned}
& C^{-}=\operatorname{diag}\left(-i \sum_{n=1}^{N} \frac{c_{n}}{\zeta-\zeta_{n}}, i \sum_{n=1}^{N} \frac{\bar{c}_{n}}{\zeta-\bar{\zeta}_{n}}\right), \\
& C^{+}=\operatorname{diag}\left(i \sum_{n=1}^{N} \frac{\bar{c}_{n}}{\zeta-\bar{\zeta}_{n}},-i \sum_{n=1}^{N} \frac{c_{n}}{\zeta-\zeta_{n}}\right) .
\end{aligned}
$$

Consider now the matrix $G_{0}$ of the form

$$
G_{0}=g_{0}^{+}(x, \zeta)-g_{0}^{-}(x, \zeta) S(\zeta) .
$$

Taking account of (42) and (75) we find that

$$
G_{0}=f_{0}^{-}(x, \zeta)\left\{-2 i \zeta^{2}[\Lambda, S(\zeta)]-C^{-} S(\zeta)+S(\zeta) C^{+}\right\} .
$$

On the other hand, according to (42) and (74) the following equality holds:

$$
\begin{aligned}
g_{0}^{+}(x, \zeta)= & g_{0}^{-}(x, \zeta) S(\zeta)+f_{0}^{-}(x, \zeta) \frac{\partial S(\zeta)}{\partial t} \\
& +\sum_{n=1}^{2 N} \Phi_{n}(x)\left[f_{n}^{+}(x, \zeta)-f_{n}^{-}(x, \zeta) S(\zeta)\right]
\end{aligned}
$$

Using equalities (42) and (73) one can easily verify that at any $\zeta \in(-\infty, \infty)$ and $n=1, \ldots, 2 N$ the equality

$$
f_{n}^{+}(x, \zeta)-f_{n}^{-}(x, \zeta) S(\zeta)=-\int_{-\infty}^{\infty} \tilde{\Psi}_{n}(z) f_{0}^{+}(z, \zeta) d z
$$


is fulfilled. By virtue of (70) the right-hand side of this equality equals zero at any $\zeta \in(-\infty, \infty)$ and $n=1, \ldots, 2 N$. With this fact in mind equality (79) takes the form

$$
g_{0}^{+}(x, \zeta)=g_{0}^{-}(x, \zeta) S(\zeta)+f_{0}^{-}(x, \zeta) \frac{\partial S(\zeta)}{\partial t} .
$$

Hence, it follows that the quantity $G_{0}$ determined by (77) admits the representation

$$
G_{0}=f_{0}^{-}(x, \zeta) \frac{\partial S(\zeta)}{\partial t} .
$$

Comparing this expression with (78) we immediately get the evolution equation for the $S$-matrix

$$
\frac{\partial S(\zeta)}{\partial t}+2 i \zeta^{2}[\Lambda, S(\zeta)]+C^{-} S(\zeta)-S(\zeta) C^{+}=0,
$$

i.e., according to (76) we have at $\zeta \in(-\infty, \infty)$,

$$
\begin{gathered}
\frac{\partial S_{11}(\zeta)}{\partial t}-i \sum_{n=1}^{N}\left(\frac{c_{n}}{\zeta-\zeta_{n}}+\frac{\bar{c}_{n}}{\zeta-\bar{\zeta}_{n}}\right) S_{11}(\zeta)=0 \\
\frac{\partial S_{12}(\zeta)}{\partial t}+4 i \zeta^{2} S_{12}(\zeta)=\frac{\partial S_{21}(\zeta)}{\partial t}-4 i \zeta^{2} S_{21}(\zeta)=0 \\
\frac{\partial S_{22}(\zeta)}{\partial t}+i \sum_{n=1}^{N}\left(\frac{c_{n}}{\zeta-\zeta_{n}}+\frac{\bar{c}_{n}}{\zeta-\bar{\zeta}_{n}}\right) S_{22}(\zeta)=0
\end{gathered}
$$

Hence, it follows that the functions $S_{11}(\zeta)$ and $S_{22}(\zeta)$ admit the representation

$$
S_{11}(\zeta)=S_{1}(\zeta) \prod_{n=1}^{N} \frac{\zeta-\zeta_{n}}{\zeta-\bar{\zeta}_{n}}, \quad S_{22}(\zeta)=S_{2}(\zeta) \prod_{n=1}^{N} \frac{\zeta-\bar{\zeta}_{n}}{\zeta-\zeta_{n}},
$$

where the quantities $S_{1}(\zeta)$ and $S_{2}(\zeta)$ are independent of time, and the points $\zeta=\zeta_{n}$ of the discret spectrum satisfy the condtion

$$
\frac{d \zeta_{n}}{d t}+i c_{n}=0, \quad n=1, \ldots, N .
$$

By virtue of (8), (10), (70), and (71), Eqs. (9) and (81) are equivalent to each other.

\section{Evolution Equations for the Normalisation Constants in the Case of a Source Satisfying the Conditions (8)}

Let $\sigma_{r}^{-}$and $\sigma_{r}^{+}$be vector-columns formed, respectively, by the elements of the $r^{\text {th }}$ column of the matrices $f_{0}^{-}$and $f_{0}^{+}$of the form (29), $r=1,2$. Then, let $\tau_{r}^{-}$and $\tau_{r}^{+}$be vector-columns formed, respectively, by the elements of the $r^{\text {th }}$ column of the matrices $g_{0}^{-}$and $g_{0}^{+}$of the form (74), $r=1$, 2. Finally, let $\zeta=\zeta_{m}$ be zeros of the function $S_{11}(\zeta)$ in the upper half-plane $\operatorname{Im} \zeta>0, m=1, \ldots, N$. According to equalities (44) and (45) we assume that at $m=1, \ldots, N$,

$$
G_{m}=\tau_{1}^{+}\left(x, \zeta_{m}\right)-B_{m} \tau_{2}^{-}\left(x, \zeta_{m}\right), \quad \hat{G}_{m}=\tau_{2}^{+}\left(x, \bar{\zeta}_{m}\right)-\hat{B}_{m} \tau_{1}^{-}\left(x, \bar{\zeta}_{m}\right) .
$$


According to equalities (75) and (76) we have

$$
\begin{aligned}
& \tau_{1}^{+}\left(x, \zeta_{m}\right)=\left(2 i \zeta_{m}^{2}+i \sum_{n=1}^{N} \frac{\bar{c}_{n}}{\zeta_{m}-\bar{\zeta}_{n}}\right) \sigma_{1}^{+}\left(x, \zeta_{m}\right), \\
& \tau_{2}^{-}\left(x, \zeta_{m}\right)=-\left(2 i \zeta_{m}^{2}-i \sum_{n=1}^{N} \frac{\bar{c}_{n}}{\zeta_{m}-\bar{\zeta}_{n}}\right) \sigma_{2}^{-}\left(x, \zeta_{m}\right), \\
& \tau_{2}^{+}\left(x, \bar{\zeta}_{m}\right)=-\left(2 i \bar{\zeta}_{m}^{2}+i \sum_{n=1}^{N} \frac{c_{n}}{\bar{\zeta}_{m}-\zeta_{n}}\right) \sigma_{2}^{+}\left(x, \bar{\zeta}_{m}\right), \\
& \tau_{1}^{-}\left(x, \bar{\zeta}_{m}\right)=\left(2 i \bar{\zeta}_{m}^{2}-i \sum_{n=1}^{N} \frac{c_{n}}{\bar{\zeta}_{m}-\zeta_{n}}\right) \sigma_{1}^{-}\left(x, \bar{\zeta}_{m}\right) .
\end{aligned}
$$

With equalities (44) and (45) taken into account it follows that the quantities $G_{m}$ and $\hat{G}_{m}$ determined by (82) can be written as follows:

$$
G_{m}=4 i \zeta_{m}^{2} B_{m} \sigma_{2}^{-}\left(x, \zeta_{m}\right), \quad \hat{G}_{m}=-4 i \bar{\zeta}_{m}^{2} \hat{B}_{m} \sigma_{1}^{-}\left(x, \bar{\zeta}_{m}\right)
$$

On the other hand, by virtue of (44), (45), and (81) the equalities

$$
\begin{aligned}
& \frac{\partial \sigma_{1}^{+}\left(x, \zeta_{m}\right)}{\partial t}=B_{m} \frac{\partial \sigma_{2}^{-}\left(x, \zeta_{m}\right)}{\partial t}+\frac{\partial B_{m}}{\partial t} \sigma_{2}^{-}\left(x, \zeta_{m}\right)+i c_{m} \chi_{m}(x), \\
& \frac{\partial \sigma_{2}^{+}\left(x, \bar{\zeta}_{m}\right)}{\partial t}=\hat{B}_{m} \frac{\partial \sigma_{1}^{-}\left(x, \bar{\zeta}_{m}\right)}{\partial t}+\frac{\partial \hat{B}_{m}}{\partial t} \sigma_{1}^{-}\left(x, \bar{\zeta}_{m}\right)-i \bar{c}_{m} \hat{\chi}_{m}(x)
\end{aligned}
$$

hold where

$$
\begin{aligned}
& \chi_{m}(x)=\left.\frac{\partial}{\partial \zeta}\left[\sigma_{1}^{+}(x, \zeta)-B_{m} \sigma_{2}^{-}(x, \zeta)\right]\right|_{\zeta=\zeta_{m}}, \\
& \hat{\chi}_{m}(x)=\left.\frac{\partial}{\partial \zeta}\left[\sigma_{2}^{+}(x, \zeta)-\hat{B}_{m} \sigma_{1}^{-}(x, \zeta)\right]\right|_{\zeta=\bar{\zeta}_{m}} .
\end{aligned}
$$

Using these equalities, from (44), (45), (73), and (74) we find that there hold relations

$$
\begin{aligned}
\tau_{1}^{+}\left(x, \zeta_{m}\right)= & B_{m} \tau_{2}^{-}\left(x, \zeta_{m}\right)+\frac{\partial B_{m}}{\partial t} \sigma_{2}^{-}\left(x, \zeta_{m}\right)+i c_{m} \chi_{m}(x) \\
& -B_{m} \sum_{n=1}^{2 N} \Phi_{n}(x) \int_{-\infty}^{\infty} \tilde{\Psi}_{n}(z) \sigma_{2}^{-}\left(z, \zeta_{m}\right) d z \\
\tau_{2}^{+}\left(x, \bar{\zeta}_{m}\right)= & \hat{B}_{m} \tau_{1}^{-}\left(x, \bar{\zeta}_{m}\right)+\frac{\partial \hat{B}_{m}}{\partial t} \sigma_{1}^{-}\left(x, \bar{\zeta}_{m}\right)-i \bar{c}_{m} \hat{\chi}_{m}(x) \\
& -\hat{B}_{m} \sum_{n=1}^{2 N} \Phi_{n}(x) \int_{-\infty}^{\infty} \tilde{\Psi}_{n}(z) \sigma_{1}^{-}\left(z, \bar{\zeta}_{m}\right) d z
\end{aligned}
$$


According to (70) at $m=1, \ldots, N$ the following equalities are fulfilled:

$$
\begin{aligned}
& \int_{-\infty}^{\infty} \tilde{\Psi}_{n}(z) \sigma_{2}^{-}\left(z, \zeta_{m}\right) d z=0, \quad \text { if } \quad n \neq m, \\
& \int_{-\infty}^{\infty} \tilde{\Psi}_{n}(z) \sigma_{1}^{-}\left(z, \bar{\zeta}_{m}\right) d z=0, \quad \text { if } \quad n=N+m .
\end{aligned}
$$

Moreover, in accordance with (43) and (70) at $m=1, \ldots, N$ the equalities

$$
\begin{gathered}
\int_{-\infty}^{\infty} \tilde{\Psi}_{m}(z) \sigma_{2}^{-}\left(z, \zeta_{m}\right) d z=i c_{m} S_{11}^{\prime}\left(\zeta_{m}\right), \\
\int_{-\infty}^{\infty} \tilde{\Psi}_{N+m}(z) \sigma_{1}^{-}\left(z, \bar{\zeta}_{m}\right) d z=-i \bar{c}_{m} S_{22}^{\prime}\left(\bar{\zeta}_{m}\right)
\end{gathered}
$$

are valid. By taking account of these equalities relation (85) becomes

$$
\begin{aligned}
\tau_{1}^{+}\left(x, \zeta_{m}\right)= & B_{m} \tau_{2}^{-}\left(x, \zeta_{m}\right)+\frac{\partial B_{m}}{\partial t} \sigma_{2}^{-}\left(x, \zeta_{m}\right) \\
& +i c_{m}\left[\chi_{m}(x)-B_{m} S_{11}^{\prime}\left(\zeta_{m}\right) \Phi_{m}(x)\right], \\
\tau_{2}^{+}\left(x, \bar{\zeta}_{m}\right)= & \hat{B}_{m} \tau_{1}^{-}\left(x, \bar{\zeta}_{m}\right)+\frac{\partial \hat{B}_{m}}{\partial t} \sigma_{1}^{-}\left(x, \bar{\zeta}_{m}\right) \\
& -i \bar{c}_{m}\left[\hat{\chi}_{m}(x)-\hat{B}_{m} S_{22}^{\prime}\left(\bar{\zeta}_{m}\right) \Phi_{N+m}(x)\right] .
\end{aligned}
$$

The quantities $\chi_{m}(x)$ and $\hat{\chi}_{m}(x)$ determined by (84) by virtue of (47), (70), and (71) admit the representation

$$
\chi_{m}(x)=a_{m} \Phi_{m}(x)+b_{m} \sigma_{1}^{+}\left(x, \zeta_{m}\right), \quad \hat{\chi}_{m}(x)=\hat{a}_{m} \Phi_{N+m}(x)+\hat{b}_{m} \sigma_{2}^{+}\left(x, \bar{\zeta}_{m}\right),
$$

where the quantities $a_{m}, b_{m}$, and $\hat{a}_{m}, \hat{b}_{m}$ are independent of $x$. With the help of (47), (49), (50), (72), and (84) we easily find that

$$
a_{m}=B_{m} S_{11}^{\prime}\left(\zeta_{m}\right), \quad \hat{a}_{m}=\hat{B}_{m} S_{22}^{\prime}\left(\bar{\zeta}_{m}\right),
$$

and $b_{m}$ and $\hat{b}_{m}$ are arbitrary quantities satisfying the condition $\hat{b}_{m}=\bar{b}_{m}, m=$ $1, \ldots, N$. Hence, it follows that the quantities $G_{m}$ and $\hat{G}_{m}$ determined by (82) can be represented in the form

$$
G_{m}=\left(\frac{\partial B_{m}}{\partial t}+i b_{m} c_{m} B_{m}\right) \sigma_{2}^{-}\left(x, \zeta_{m}\right), \quad \hat{G}_{m}=\left(\frac{\partial \hat{B}_{m}}{\partial t}-i \hat{b}_{m} \bar{c}_{m} \hat{B}_{m}\right) \sigma_{1}^{-}\left(x, \bar{\zeta}_{m}\right) .
$$

Comparing these equalities with (83) we get that the evolution equations for the normalisation constants $B_{m}$ and $\hat{B}_{m}$ have the form

$$
\frac{\partial B_{m}}{\partial t}-i\left(4 \zeta_{m}^{2}-b_{m} c_{m}\right) B_{m}=0, \quad \frac{\partial \hat{B}_{m}}{\partial t}+i\left(4 \bar{\zeta}_{m}^{2}-\hat{b}_{m} \bar{c}_{m}\right) \hat{B}_{m}=0 .
$$

On the basis of the equality $\hat{b}_{m}=\bar{b}_{m}$ the system of equations (86) has an invariant manifold $\hat{B}_{m}=-\bar{B}_{m}$. 
Thus, for a source satisfying the conditions (8) the system of equations (80) and (86) describes the evolution in time of all scattering data for the operator $L$ of the form (6), (7) with the potential $u=u(x, t)$ satisfying the system (1)-(3). This means that for solving the Cauchy problem for the system (1)-(3) we can also use the inverse scattering method for the operator $L$ of the form (6), (7).

\section{Conclusion}

In conclusion, we should like to note that for a source satisfying the conditions (5) the system (1)-(3) has a one-soliton solution of the form

$$
\begin{aligned}
u & =a \frac{\exp \left[2 i \bar{\zeta}_{0}(x-f)\right]}{1+\exp [4 \mu(x-f)]}, \\
\varphi_{n} & =-\frac{i}{2} a \beta_{n} \frac{\exp \left[-i\left(\zeta_{n}-2 \bar{\zeta}_{0}\right)(x-f)\right]}{1+\exp [4 \mu(x-f)]}, \\
\psi_{n} & =\frac{1+\alpha_{n} \exp [4 \mu(x-f)]}{1+\exp [4 \mu(x-f)]} \exp \left[-i \zeta_{n}(x-f)\right], \\
p_{n} & =c_{n} \frac{\alpha_{n}+\exp [4 \mu(x-f)]}{1+\exp [4 \mu(x-f)]} \exp \left[i \zeta_{n}(x-f)\right], \\
q_{n} & =-\frac{i}{2} \bar{a} \beta_{n} c_{n} \frac{\exp \left[i\left(\zeta_{n}-2 \zeta_{0}\right)(x-f)\right]}{1+\exp [4 \mu(x-f)]}, \quad n=1, \ldots, N,
\end{aligned}
$$

where $c_{n}$ and $\zeta_{n}$ are arbitrary complex-valued functions of time satisfying the only condition $\operatorname{Im} \zeta_{n}>0, n=1, \ldots, N$, the quantity $\zeta_{0}$ is independent of time and has the form $\zeta_{0}=i \mu+v, \mu>0, v \in(-\infty, \infty)$, and the quantities $\alpha_{n}$ and $\beta_{n}$ are determined by the equalities

$$
\alpha_{n}=\frac{\zeta_{n}-\zeta_{0}}{\zeta_{n}-\bar{\zeta}_{0}}, \quad \beta_{n}=\frac{1}{\zeta_{n}-\bar{\zeta}_{0}}, \quad n=1, \ldots, N .
$$

In this case, the quantities $a$ and $f$ are time functions satisfying the equations

$$
\frac{i}{a} \frac{d a}{d t}+4\left(\mu^{2}+v^{2}\right)=F+\frac{v}{\mu} G, \quad 2 \mu\left(\frac{d f}{d t}-4 v\right)+G=0,
$$

where $F$ and $G$ equal, respectively, the real and imaginary parts of the quantity $H$ of the form

$$
H=\sum_{n=1}^{N}\left(\alpha_{n} \beta_{n} c_{n}+\bar{\beta}_{n} \bar{c}_{n}\right)
$$

Moreover, the quantity $a$ satisfies the condition $|a|=4 \mu$ that, obviously, does not contradict Eqs. (88). One can easily see that choosing properly the quantities $c_{n}$ and $\zeta_{n}, n=1, \ldots, N$, we can make soliton (87) to have an a priori given motion. However, an absolute value of the amplitude $a$ will be conserved in time. A detailed analysis shows that in this case a multi-soliton solution of the system (1)-(3), describing the interaction of several solitons of the form (87), may have a nontrivial dynamics. In particular, it can describe the decay and fusion of solitons, the capture of solitons into the oscillatory regime of motion, and the formation of a bound state of several solitons. 
Then, for a source satisfying the conditions (8), a one-soliton solution of the system (1)-(3) has the form

$$
\begin{aligned}
u & =a \frac{\exp [2 i \bar{\zeta}(x-f)]}{1+\exp [4 \mu(x-f)]}, \\
\varphi & =2 i \mu W \frac{\exp [4 \mu(x-f)]}{1+\exp [4 \mu(x-f)]} \exp [i \zeta(x-f)], \\
\psi & =c \frac{\exp [-i \zeta(x-f)]}{1+\exp [4 \mu(x-f)]}, \\
p & =\frac{i}{c}\left\{\left[\frac{1}{2 \mu}-2(x-f)\right] \varphi+i W \frac{\exp [i \zeta(x-f)]}{1+\exp [4 \mu(x-f)]}\right\}, \\
q & =-\frac{i}{c}\left\{\left[\frac{1}{2 \mu}+2(x-f)\right] \psi+\frac{c}{2 \mu} \frac{\exp [4 \mu(x-f)]}{1+\exp [4 \mu(x-f)]} \exp [-i \zeta(x-f)]\right\},
\end{aligned}
$$

where $W$ is an arbitrary complex-valued function of time, the quantity $\zeta$ has the form $\zeta=i \mu+v, \mu \geq 0, v \in(-\infty, \infty)$, and the quantity $c$ is determined from the relation $i a c=8 \mu^{2} W$. In this case, the quantities $\zeta, f$ and $a$ satisfy the equations

$$
\begin{gathered}
\frac{d \zeta}{d t}=i W, \quad \frac{d f}{d t}=4 v, \\
\frac{d a}{d t}=\left[4 i\left(\mu^{2}+v^{2}\right)+\frac{1}{2 \mu}(W+\bar{W})\right] a .
\end{gathered}
$$

Moreover, the quantity $a$ satisfies the condition $|a|=4 \mu$ that, as one can easily verify, does not contradict equations (90). Due to the equality $v=\operatorname{Re} \zeta$, under a proper choice of the function $W$ we can make soliton (89) to have an a priori given motion. However, if at the time moment $t=t^{\prime}$ the point $\zeta$ reaches the real axis, i.e., the quantity $\mu$ vanishes, the quantity $a$ also vanishes. Thus, we find that $u\left(x, t^{\prime}\right) \equiv 0$, i.e., at the time moment $t=t^{\prime}$ the obtained soliton disappears. When at $t>t^{\prime}$ the quantity $\zeta$ leaves the real axis, the soliton considered appears again. The multi-soliton solution of the system (1)-(3), obtained by the inverse scattering method, describes in this case the interaction of several solitons of the form (89). It has a rich enough dynamics. To investigate the interaction of solitons of the form (87) and (89) with each other, we should take in Eq. (1) a source formed by solutions of the system (2), (3) some of which satisfy the conditions (5) and the others satisfy the conditions (8). One should remember that an additive nature of a source composition corresponds to a multiplicative nature of composition of scattering data.

\section{References}

1. Zakharov, V.E., Shabat, A.B.:Exact theory of two-dimensional self-focussing and one-dimensional self-modulation of waves in nonlinear media. Sov. Phys. JETP 34, 62 (1972)

2. Mel'nikov, V.K.: JINR Preprint P2-88-668, Dubna 1988

3. Mel'nikov, V.K.: JINR Preprint P2-88-728, Dubna 1988 
